\title{
Vulnerabilidad de los servicios ecosistémicos del área marina protegida Uramba
}

\section{Ecosystems services vulnerability of Uramba Marine Protected Area}

\author{
Natalia Uribe-Castañeda ${ }^{1,2 *}$, Carlos Andrés Satizabal ${ }^{l}$, Leonardo Herrera Orozco ${ }^{3}$ y Jaime Ricardo Cantera Kintz ${ }^{l}$ \\ $\begin{array}{llll}\text { (iD) } 0000-0002-3797-5894 & \text { (D) } 0000-0003-1791-5904 & \text { (D) } 0000-0001-9851-8606 & \text { (ID } 0000-0002-4010-298 X\end{array}$
}

1. Ecomanglares Research Group of Estuaries and Mangrove Ecology, Biology Department, Faculty of Natural and Exact Sciences, University of Valle, Cali,Colombia.natalia.uribe@correounivalle.edu.co*, casatiza@gmail.com, jaime.cantera@correounivalle.edu.co

2. Interdisciplinary Ecology Program, School of Natural Resources and Environment, University of Florida, Gainesville, Florida.

3. Natura Group, Biological Science Department, ICESI University, Cali, Colombia.lherrera@icesi.edu.co

* Autora de correspondencia

\section{RESUMEN}

$\mathrm{L}$ os sistemas marinos y costeros del área marina protegida (AMP) Uramba, así como en el resto del mundo, proporcionan una gran variedad de servicios ecosistémicos (i. e. protección costera, regulación de ciclos geoquímicos o provisión de alimento, etc.). Amenazas naturales y/o antropogénicas (i. e. como la erosión costera, el aumento relativo en el nivel del mar o la sobrepesca) pueden transformar, degradar o limitar el suministro de estos servicios ecosistémicos. Este estudio explora el grado de vulnerabilidad que presentan los servicios ecosistémicos del AMP Uramba ante las amenazas naturales y antropogénicas presentes. Los métodos incluyen una revisión bibliográfica, un taller de expertos, trabajo de campo para la validación y la georreferenciación de servicios y amenazas. Para calcular la vulnerabilidad de los servicios ecosistémicos, se estimó la magnitud de las amenazas, su riesgo y su capacidad de resiliencia. Las amenazas naturales y antropogénicas más frecuentes se encuentran en la región externa del AMP Uramba, en las localidades de Juanchaco, Ladrilleros y Base Naval. El aumento en el nivel del mar es la amenaza que más podría vulnerar los servicios ecosistémicos del AMP Uramba, incluyendo los siguientes: protección costera en playas arenosas, planos lodosos y manglares; regulación climática en playas arenosas y planos lodosos; recreación y turismo en playas arenosas y manglares; mantenimiento de ciclos de vida en manglares, y provisión de alimentos en planos lodosos. Los servicios ecosistémicos de la costa rocosa están amenazados principalmente por deslizamientos, y en el ecosistema pelágico por la sobrepesca.

PALABRAS CLAVE: Amenazas, resiliencia, riesgo, Pacífico colombiano, Pacífico Oriental Tropical

\section{ABSTRACT}

$\mathrm{S}$ ystems of Uramba Marine Protected Area (MPA), like marine and coastal systems worldwide, sustain a great variety of ecosystem services (ES) (i.e. coastal protection, climate regulation, or food provision, etc.). Anthropogenic and natural stressors (i.e. coastal erosion, sea level rise, or overfishing, etc.) can transform, degrade, or limit the supply of ecosystem services. This study explores the vulnerability of Uramba MPA ecosystem services to anthropogenic and natural stressors. Stressors and ecosystems services were characterized by a literature review, an expert's workshop, fieldtrips for validation, and georeferencing. To calculate the vulnerability of ecosystem services, the magnitude of each stressor, their risks, and their resilience capacity was estimated. Natural and anthropogenic stressors are more frequent at the outer region of Uramba MPA, in Ladrilleros, Juanchaco, and Base Naval. Sea level rise is the main stressor that potentially will affect Uramba MPA ecosystems services, including the following: coastal protection in sandy beaches, mud flats, and mangroves; climate regulation in sandy beaches and mud flats; recreation and tourism in sandy beaches and mangroves; life cycle maintenance in mangroves, and food provision in mud flats. Ecosystems services in rocky coasts are threatened by landslides, and in pelagic systems by overfishing.

KEYWORDS: Stressors, resilience, risk, Colombian Pacific, Tropical Eastern Pacific

DOI: https://doi.org/10.25268/bimc.invemar.2020.49.SuplEsp.1055

Publicado por INVEMAR

Published by INVEMAR

Este es un manuscrito de acceso abierto bajo la licencia CC

Reconocimiento-No Comercial-Compartir Igual 


\section{INTRODUCCIÓN}

La Evaluación de Ecosistemas del Milenio generó una visión holística del valor de la biosfera, de importancia global, regional y local. Esta evaluación tiene en cuenta aspectos ecológicos, socioeconómicos y culturales de los Servicios de los Ecosistemas (SE) (Agardy et al., 2005). El concepto de SE ha ganado atención entre científicos, ambientalistas y actores políticos en los últimos años. El principio básico de los SE es brindar una herramienta para integrar componentes sociales, económicos y ambientales que proveen los ecosistemas, y de esta manera aconsejar la toma de decisiones estratégicas. Los SE muestran de forma efectiva los beneficios que los ecosistemas brindan a los humanos y sus valores (monetarios y no monetarios) (De Groot et al., 2010; Costanza et al., 2014).

Liquete et al. (2013) realizaron una revisión de la literatura centrada en los SE marinos y costeros, y encontraron que los SE más comunes estudiados son el suministro de alimentos, la purificación de agua, la protección costera, la recreación y el turismo. Identificaron, además, una brecha de conocimiento en la disponibilidad de indicadores que midan la capacidad y el flujo o beneficio de cada SE.

La identificación y valoración de los $\mathrm{SE}$ ha contribuido significativamente a comunicar al público las razones por las que se debe conservar la biodiversidad y por qué el uso de los recursos marinos debe llevarse a cabo de manera sostenible. Los ecosistemas marinos y costeros del Pacífico Oriental Tropical están siendo cada vez más vulnerados por amenazas naturales y antropogénicas como el cambio climático, cambios en el uso del hábitat, sobreexplotación de recursos y contaminación (Backer et al., 2010). El estudio de los SE permite definir las opciones de gestión con mayor precisión; enfrentar las deficiencias ambientales, políticas, sociales y administrativas; guiar a los tomadores de decisiones y a las comunidades hacia la sostenibilidad y, con suerte, la conservación (Cork, 2010). La Evaluación de Ecosistemas del Milenio destacó la importancia de los ecosistemas para los seres humanos y presentó el estado actual de los SE en todo el mundo. Según esta evaluación, más de $60 \%$ de los $\mathrm{SE}$ en todo el mundo está degradado (Agardy et al., 2005). La importancia de la Evaluación de Ecosistemas ha aumentado con el desarrollo de los Objetivos de Desarrollo Sostenible (ODS) creados en 2015 para abordar varios temas en pro del bienestar humano y del medio ambiente. Diferentes trabajos han identificado importantes contribuciones de este enfoque en

\section{INTRODUCTION}

The Millennium Ecosystem Assessment provides a holistic view of the value of the biosphere, taking into account ecological, socioeconomic, and cultural aspects of Ecosystem Services (ES) that are of global, regional and local importance (Agardy et al., 2005). The concept of ES has gained increasing attention amongst scientists, environmentalists and policy makers in recent years. Its basic idea is to provide a tool to integrate social, economic and environmental components of services provided by ecosystems in order to guide strategic decision making. It is an effective way to demonstrate the benefits that ecosystems provide to humans and their values (monetary and non-monetary) (De Groot, 2010; Costanza et al., 2014).

Liquete et al. (2013) carried out a literature review focused on Marine and Coastal ES, where they found that the most common ES studied were food provision, water purification, coastal protection, and recreation and tourism. They identified knowledge gaps regarding the availability of indicators that measure the capacity, flow or benefit derived from each ES.

Identifying and valuing ES has significantly contributed to our understanding of the reasons why we must conserve biodiversity, and why the use of marine resources must be carried out sustainably. Habitat change, climate change, over-exploitation and pollution are becoming more acute and frequent in marine and coastal environments, such as in the Tropical Eastern Pacific (Backer et al., 2010). The study of ES allows us to: define management choices more accurately; confront environmental, political, social and administrative shortcomings; guide decision makers and communities towards sustainability and, hopefully, conservation (Cork, 2010). The Millennium Ecosystem Assessment highlighted the importance of ecosystems to humans, and furthermore presented the current state of ES worldwide. The importance of the Ecosystem Assessment has increased with the development of the Sustainable Development Goals (SDGs), which were created in 2015 to address several issues for human welfare and the environment. Different works have identified important contributions of this approach in the development of plans to achieve the goals set out in about 12 of the 17 SDGs (McCartney et al., 2015; Wood et al., 2018; Veidemane, 2019). This research represents a contribution towards SDG $13^{\text {th }}$ Climate Action 
el desarrollo de planes para alcanzar las metas planteadas en aproximadamente 12 de los 17 ODS (McCartney et al., 2015; Wood et al., 2018; Veidemane, 2019). Esta investigación representa una contribución al ODS 13 de Acción Climática y el ODS 14 de Vida Submarina.

Cantera et al. (2013) evaluaron las condiciones ecológicas, ambientales y socioeconómicas del AMP Uramba, así como los desafíos para la conservación de los servicios ecosistémicos en la región. Este contexto socioecológico se ha tenido en cuenta para establecer un marco metodológico interdisciplinario para la vulnerabilidad. En esta investigación definimos el termino "vulnerabilidad" como el grado en que un sistema experimenta daño debido a la exposición a una perturbación o una amenaza (Turner II et al., 2003; Mumby et al., 2014; Weißhuhn et al., 2018). La evaluación de la vulnerabilidad centrada en amenazas ha demostrado ser insuficiente para comprender las respuestas de un sistema afectado. Una comprensión más compleja debería incluir el riesgo y la resiliencia ante las amenazas. El riesgo es una medida de la probabilidad de que ocurra un evento y la magnitud de sus consecuencias. En esta investigación se adopta la definición de resiliencia ecológica de Walker et al. (en Spears et al., 2015), esto es, "la capacidad de un sistema para absorber perturbaciones y reorganizarse mientras mantiene sus funciones, estructura, identidad y respuestas". Es la capacidad de un sistema para mantener o recuperar ciertas funciones a pesar de las perturbaciones (Turner II et al., 2003).

Las evaluaciones de vulnerabilidaddelos ecosistemas se han identificado como un enfoque válido para abordar la gestión de amenazas (Oppenheimer et al., 2014; Weißhuhn et al., 2018). Combinar el estudio de la vulnerabilidad del hábitat con la identificación de los servicios ecosistémicos es útil para determinar acciones y evitar que ocurran eventos estresantes (prevención), o al menos aliviar sus efectos (adaptación) (Schröter et al, 2004). La resiliencia ecológica es la capacidad de un ecosistema de mantener sus funciones ecológicas ante amenazas, y los umbrales ecológicos representan los límites donde los cambios en las condiciones ambientales producidos por amenazas conducen a cambios en el estado de los ecosistemas (Sasaki et al., 2015). Estudios basados en la resiliencia ecológica y los umbrales ecológicos de un ecosistema representan una guía para los tomadores de decisiones (Spears et al., 2015; Weißhuhn et al., 2018).

El AMP Uramba presenta una amplia variedad de ecosistemas, lo que genera un desafío para la toma de decisiones sobre qué ecosistemas y qué servicios deben and $14^{\text {th }}$ Life below Water. According to this assessment, over $60 \%$ of ES worldwide are degraded (Agardy et al., 2005). Therefore, it is mandatory to call attention to the state of ES and research associated with the conservation of ecosystems and their services.

Cantera et al. (2013) presented a description of the ecological, environmental and socioeconomic conditions of Uramba MPA, as well as the challenges for the conservation of ecosystem services in the region, a task in which all stakeholders should be integrated. This social-ecological context adds to the different systematic attempts to establish an interdisciplinary methodological framework for vulnerability, which we define in this paper as the degree to which a system or some component is likely to experience harm due to exposure to a perturbation or stressor (Turner II et al., 2003; Mumby et al., 2014; Weißhuhn et al., 2018). Vulnerability assessment focused on stressors has proven to be insufficient for understanding the responses of an affected system. A more complex understanding should include risk and resilience. Risk is a measure of the likelihood of the occurrence of an event, and the magnitude of its consequences. Despite the different meanings of ecological resilience, here we take the definition by Walker et al. (in Spears et al., 2015), that is "the capacity of a system to absorb disturbance and reorganize while undergoing change so as to retain essentially the same function, structure, identity and feedbacks"; it means the capacity of a system to maintain or recover certain functions despite disturbance (Turner II et al., 2003).

Ecosystem vulnerability assessments have been identified as a valid approach to address the management of threats (Oppenheimer et al., 2014; Weißhuhn et al., 2018). Coupling the study of habitat vulnerability with the identification of ecosystem services can be useful to determine which actions must be taken to prevent stressful events from happening (prevention), or at least to alleviate their effects (adaptation) (Schröter et al., 2004). Knowledge based on ecological resilience and ecological threshold can be used as practical guidance for policymakers (Spears et al., 2015; Weißhuhn et al., 2018). Ecological resilience is the ability to maintain ecological functions, which differ from one ecosystem to other. Ecological thresholds represent the limits where even changes in environmental conditions associated with natural and/or anthropogenic stressors lead to changes in ecosystem states (Sasaki et al., 2015). 
priorizarse. Como sugirieron Mumby et al. (2014), la vulnerabilidad es un concepto útil para áreas que representan un sistema socioecológico complejo, y es útil combinar este análisis con la resiliencia ecológica (Robertson and McGee, 2003; Hughes et al., 2005; Baho et al., 2017; Carr et al., 2017). Es por esto que establecer la resiliencia ecológica puede ser una herramienta para mitigar el impacto de diferentes amenazas y promover acciones que reduzcan la vulnerabilidad ante estas. Por lo razones, el objetivo de esta investigación fue evaluar la vulnerabilidad de los servicios ecosistémicos en el AMP Uramba, como modelo en el Pacífico Oriental Tropical. Los resultados de este análisis no solo brindan información clave para la toma de decisiones relacionadas con la administración y el manejo de áreas marinas protegidas, sino también sobre la sostenibilidad, resiliencia y adaptación a largo plazo ante los peligros naturales, los cuales son difíciles de evitar no solo en el área estudiada, sino también en otras AMP con características similares en el Pacífico Tropical Oriental.

\section{ÁREA DE ESTUDIO}

El AMP Uramba $\left(3^{\circ} 56^{\prime}\right.$ a $4^{\circ} 05^{\prime} \mathrm{N}$ y $77^{\circ} 19^{\prime}$ a $\left.77^{\circ} 21^{\prime} \mathrm{W}\right)$ pertenece al hotspot de biodiversidad Tumbes-Chocó-Magdalena, establecido por Conservación Internacional como una de las 36 regiones de prioridad mundial para la conservación (www.conservación.org; 20/julio/2020). En 2010, el Gobierno colombiano estableció a Uramba como área marina protegida (http://www. parquesnacionales. gov.co; 20/julio/2020) bajo la figura de protección del Parque Nacional Natural, es decir, es un área que permite la autorregulación ecológica, en la cual los ecosistemas no están siendo alterados por la explotación humana, y además tiene un valor científico, educativo, estético y recreativo en el ámbito nacional (Decreto 2811 de 1974).

El AMP Uramba tiene una extensión de aproximadamente $136 \mathrm{~km}^{2}$ de ecosistemas marinos y costeros. Estos ecosistemas se presentan en la Figura 1 e incluyen manglares, playas arenosas, planos lodosos, costas rocosas y sistemas pelágicos (Cantera et al., 1999). Cantera et al. (2013), a través de una revisión bibliográfica, establecieron la presencia de 2178 especies en ese lugar. Castellanos-Galindo et al. (2011) registró 39 especies en estado de amenaza ( 1 esponja, 5 cnidarios, 10 moluscos, 7 crustáceos y 16 peces); algunas de estas especies son económicamente importantes para las poblaciones humanas (i. e. caracol Anadara tuberculosa y Anadara similis; caracol
Uramba MPA presents a wide variety of ecosystems, which creates a challenge for decision-making regarding which ecosystems and which services should be prioritized. As suggested by Mumby et al. (2014), vulnerability is a useful concept for areas that represent a complex socio-ecological system, and it is helpful to combine this analysis with ecological resilience (Robertson and McGee, 2003; Hughes et al., 2005; Baho et al., 2017; Carr et al., 2017). This is why establishing ecological resilience can be a tool to mitigate the impact of different stressors or promote actions that reduce vulnerability. For these reasons, our main goal was to assess the vulnerability of ecosystem services in Uramba MPA, as a model in the Tropical Eastern Pacific. The results of this analysis not only provide key information for decision making related to the administration and management of marine protected areas, but it also to long-term sustainability, resilience and adaptation to natural hazards, which are difficult to avoid not only in the area studied, but also in other MPAs with similar characteristics in the Tropical Eastern Pacific.

\section{STUDY AREA}

Uramba MPA $\left(3^{\circ} 56^{\prime}\right.$ to $4^{\circ} 05^{\prime} \mathrm{N}$ and $77^{\circ} 19^{\prime}$ to $\left.77^{\circ} 21^{\prime} \mathrm{W}\right)$ belongs to the Tumbes-Chocó-Magdalena biodiversity hotspot, established by Conservation International as one of 36 global priority regions for conservancy (www.conservation.org; Search: 20-July-20). In 2010, the Colombian government established it as a Marine Protected Area (http://www.parquesnacionales. gov.co; Search: 20-July-20) under the figure of protection of National Natural Park, meaning it is an area in which its extension allows ecological autoregulation, the ecosystems are not being altered by human exploitation, and it has a scientific, educative, aesthetic, and recreative value at national level (Decree 2811 of 1974).

The MPA has an extent of approximately $136 \mathrm{~km}^{2}$ of marine and coastal ecosystems. These ecosystems are presented in Figure 1, and they include mangroves, sandy beaches, mud flats, rocky coast and pelagic systems (Cantera et al., 1999). A review by Cantera et al. (2013) indicated the presence of 2178 species in Uramba MPA. Castellanos-Galindo et al. (2011) recorded 39 species (1 sponge, 5 cnidarians, 10 molluscs, 7 crustaceans and 16 fish) in threatened status; some of these species are economically important to human populations (i.e.: conch Anadara tuberculosa and Anadara similis; snail 


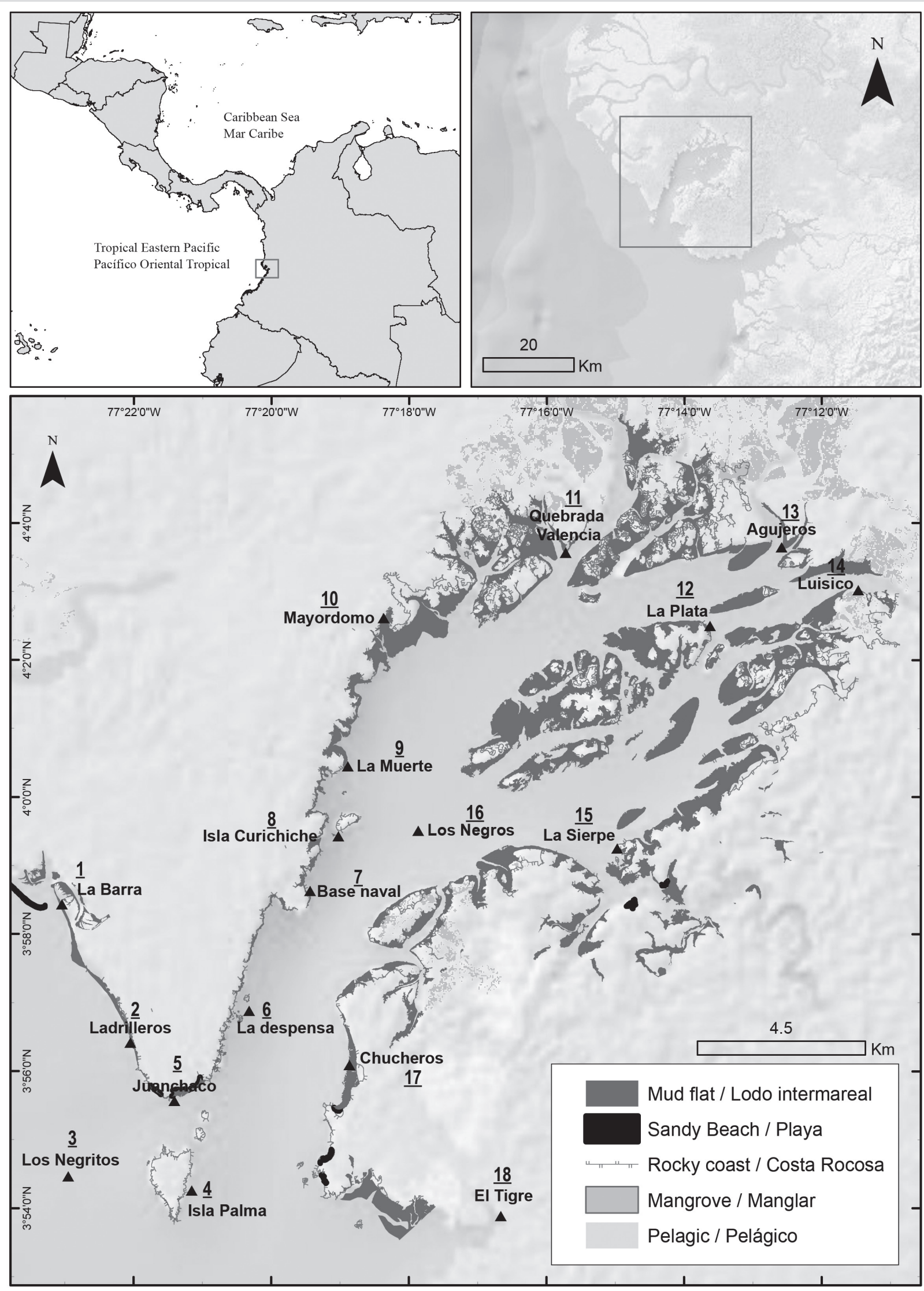

Figura 1. Mapa del AMP Uramba y las 18 localidades estudiadas.

Figure 1. Map of the Uramba MPA and the 18 studied locations. 
Titanostrombus galeatus; camarones Penaeus vannamei y Penaeus occidentalis, y mero Epinephelus itajara).

Los asentamientos humanos del AMP Uramba son principalmente afrodescendientes, y dependen de los servicios ecosistémicos que brindan los manglares, esteros y el área de selva continental que rodea la bahía. La mayoría de las comunidades que viven en el interior se encuentran dispersas en diversas áreas con baja población: La Plata (localidad 12), Luisico (localidad 14), Chucheros (localidad 17), y tienen formas de vida basadas principalmente en la pesca (salmonetes, pargos, bagres, camarones y bivalvos), caza (perezosos, mapaches y agutíes) y tala artesanal. Las comunidades de la zona exterior del AMP Uramba habitan en Juanchaco (localidad 5), Ladrilleros (localidad 2) y La Barra (localidad 1), también son afrodescendientes y obtienen su sustento principalmente del turismo de playa y el avistamiento de ballenas jorobadas. La población de estos asentamientos es de aproximadamente 30000 personas (DANE, 2012). En los últimos años, tanto los habitantes del exterior como del interior del AMP han desarrollado proyectos de ecoturismo que hacen uso no extractivo de los servicios ecosistémicos de la zona. De igual forma, hay presencia de algunas comunidades indígenas, pero estas no usan directamente los servicios ecosistémicos costeros y marinos del área, debido a que sus asentamientos se encuentran ubicados en la selva.

\section{MATERIALES Y MÉTODOS}

\section{Identificación de factores estresantes}

Se realizó una revisión de la literatura y un taller de expertos para identificar las amenazas presentes en el AMP Uramba. La literatura que se tuvo en cuenta para realizar un listado de las posibles amenazas de los ecosistemas marinos y costeros del AMP Uramba fue Invemar-Univalle-Inciva (2006), Univalle (2010) y Castellanos-Galindo et al. (2011). El taller de expertos se realizó en el marco del Curso de Manejo Integrado de Zonas Costeras en 2012, impartido por el Instituto Colombiano de Investigaciones Marinas y Costeras “José Benito Vives de Andréis". El equipo del taller de expertos estuvo conformado por expertos locales con conocimiento tradicional del AMP Uramba, funcionarios de Parques Nacionales Naturales de Colombia, funcionarios de la Corporación Autónoma Regional del Valle, investigadores del grupo de investigación Ecología de Estuarios y Manglares (con 5 a 20 años de experiencia estudiando el AMP Uramba), y profesores de la Universidad del Valle (con 20 a 45 años de experiencia estudiando esta AMP). El taller de expertos
Titanostrombus galeatus; shrimp Penaeus vannamei and Penaeus occidentalis; and grouper Epinephelus itajara).

The human settlements of the Uramba MPA are African descendants that have been sustained by the ecosystem services provided by the mangroves, estuaries, and the continental jungle area surrounding the bay. Most of the communities living in the interior are scattered in various areas with low population numbers: La Plata (location 12), Luisico (location 14), Chucheros (location 17) and have ways of life based mainly on fishing (mullets, snappers, catfish, shrimp and bivalves), hunting (sloths, raccoons, and agoutis) and artisanal logging. The communities in the outer zone of Uramba MPA, who inhabit the towns of Juanchaco (location 5), Ladrilleros (location 2), and La Barra (location 1) are also African descendants and derive their livelihood mainly from beach tourism and Humpback whale watching. The population of these settlements is approximately 30000 people (DANE, 2012). In recent years, both the inhabitants of the exterior and interior have developed ecotourism projects that make non-extractive use of the ecosystem services of all the Uramba MPA ecosystems. There are also some indigenous communities, but they only partially derive their livelihood from the coastal and marine ecosystems, due to the location of their settlements deep in the jungle.

\section{MATERIALS AND METHODS}

\section{Identification of stressors}

A literature review and an expert workshop were conducted to summarize potential stressors present in Uramba MPA. The literature taken into account were Invemar-Univalle-Inciva (2006), Univalle (2010), and Castellanos-Galindo et al. (2011). The expert's workshop was conducted in the context of the Integrated Coastal Zone Management Course in 2012, issued by the Colombian Marine and Coastal Research Institute "Jose Benito Vives de Andreis". The expert workshop team was formed by local experts with a lot of traditional knowledge of Uramba MPA, staff members from Colombian National Natural Parks, staff members of the Valle Regional Autonomous Corporation, researchers from the research group Ecology of Estuaries and Mangroves with 20 years of experience studying Uramba MPA, and professors from the University of Valle with more than 45 years of experience studying Uramba MPA. The expert's workshop was conducted using participatory tools. The experts participated in a story 
se llevó a cabo utilizando herramientas participativas. Los expertos participaron en una sección de mapas parlantes, en la que se identificaron las amenazas del AMP Uramba. Tanto los datos obtenidos en los mapas como en la revisión de la literatura fueron validados y caracterizados espacialmente a través de recorridos en bote alrededor del lugar.

Estimación de la vulnerabilidad de los servicios ecosistémicos

Primero, se identificaron los tipos de ecosistemas costeros presentes en el AMP Uramba: costa rocosa, manglares, planos lodosos, playas arenosas y sistemas pelágicos. En cada ecosistema, se identificaron los servicios ecosistémicos con base en su representatividad y disponibilidad en el área.

Los servicios ecosistémicos del AMP Uramba se caracterizaron utilizando como contexto la literatura existente disponible. El concepto fue reestablecido a finales de la década de 1970 (Schumacher, 1973) y puesto en la agenda de la investigación de ecosistemas al final del siglo pasado (Costanza et al., 1997; Daily, 1997; Mooney y Ehrlich, 1997), lo que llevó al auge de la investigación de los servicios de los ecosistemas en la década del 2000 (Agardy et al., 2005; Boyd y Banzhaf, 2007; Costanza et al., 2014, 2017). Para clasificarlos, se tomó en cuenta la clasificación de la revisión sistemática para los sistemas marinos y costeros desarrollada por Liquete et al. (2013).

Investigadores del grupo de investigación Ecología de Estuarios y Manglares y profesores de la Sección de Biología Marina de la Universidad del Valle utilizaron los resultados de la revisión de la literatura, los mapas parlantes y los recorridos de validación en barco para seleccionar y estimar la magnitud de las amenazas que podrían vulnerar los servicios ecosistémicos de cada ecosistema. Los profesores e investigadores también estimaron el riesgo y la resiliencia. La magnitud de las amenazas se estimó utilizando la escala espacial propuesta por Halpern et al. (2008), donde los factores de estrés se calificaron como altos (3) cuando afectaban a nivel del Pacífico Oriental Tropical; moderada (2) cuando afectaban el AMP; y baja (1) cuando afectaban solo la localidad donde se encontraban. La probabilidad de ocurrencia de cada estresor y sus consecuencias (riesgo), así como la capacidad de un ecosistema para recuperar sus servicios y funciones ecológicas (resiliencia) se estimaron como mínimos $(0,2)$, bajos $(0,4)$, moderados $(0,6)$, altos $(0,8)$ o máximos (1). El riesgo se determinó como máximo (1) cuando la probabilidad de ocurrencia era muy alta $y$ map section, in which they identified the Uramba MPA stressors in a map. Both the data obtained from the story map and the literature review were validated and spatially characterized in the field through boat trips around the entire Uramba MPA.

\section{Estimation of ecosystem services vulnerability}

First, the five coastal types of ecosystems present in Uramba MPA were identified: rocky coast, mangroves, mud flats, sandy beaches and pelagic systems. In each ecosystem, relevant ecosystem services were identified based on knowledge of their representativeness and availability in the area.

The ecosystem services of the Uramba MPA were characterized using as context the extent literature available regarding ecosystem services. The concept was re-established in the late 1970s (Schumacher, 1973), and was put on the agenda of ecosystem research at the end of the last century (Costanza et al., 1997; Daily, 1997; Mooney and Ehrlich, 1997), leading to the boom of ecosystem services research in the 2000s (Agardy et al., 2005; Boyd and Banzhaf, 2007; Costanza et al., 2014, 2017). The systematic review classification for marine and coastal systems developed by Liquete et al. (2013) were taken into account to classify them.

Researchers from the research group Ecology of Estuaries and Mangroves and professors of the Marine Biology section at the University of Valle used the results from the literature review, the talking maps, the boat trips to select and estimate the magnitude of the stressors that could vulnerate the ecosystem services of each coastal ecosystem. Professors and researchers also estimated risk and resilience. The magnitude of the stressors was evaluated using the spatial scale proposed by Halpern et al. (2008), where stressors were rated as high (3) when they affected the Tropical Eastern Pacific region; moderate (2) when they affected the entire MPA; and low (1) when they affected only the location where they were encountered. The probability of occurrence of each stressor and its consequences (risk), and the ability of an ecosystem to recover its services (resilience) were estimated as minimum (0.2), low (0.4), moderate (0.6), high (0.8), and maximum (1). The risk was determined as maximum (1) when the probability of occurrence was very high, and minimum (0.2) when the probability was very low. The resilience was determined as maximum (1) when the ability of the ecosystem to recover was very 
como mínimo $(0,2)$ cuando la probabilidad era muy baja. La resiliencia se determinó como máxima (1) cuando la capacidad de recuperación del ecosistema era muy alta y como mínima $(0,2)$ cuando la probabilidad era muy baja.

La vulnerabilidad se determinó como una función matemática de la magnitud de la amenaza, multiplicada por el riesgo (probabilidad de ocurrencia y sus consecuencias) y dividida por la resiliencia (capacidad de recuperación del ecosistema) (Kaly et al., 1999; Gowrie, 2003; Turner II et al., 2003; Zacharias y Gregr, 2005).

$\begin{aligned} & \text { Vulnerabilidad del } \\ & \text { servicio del ecosistema }\end{aligned}=\frac{\text { Factor de estrés } \times \begin{array}{l}\text { Riesgo del servicio } \\ \text { del ecosistema }\end{array}}{\text { Resiliencia }}$

Por ejemplo, para el ecosistema de planos lodosos, calculamos la vulnerabilidad de la provisión de alimentos ante la amenaza "aumento del nivel del mar" de la siguiente manera: amenaza, con el valor máximo de 3; riesgo, con el valor máximo de 1 , y resiliencia, con el valor mínimo de 0,2 .

$$
\begin{aligned}
& \text { Vulnerabilidad en el } \\
& \text { suministro de alimentos }
\end{aligned}=\frac{3 \times 1}{0,2}=15
$$

Debido a la superposición en los rangos de valores obtenidos, modulados principalmente por la magnitud del estresante, se generó un modelo de color para facilitar su interpretación. Se utilizó el verde para representar la mínima vulnerabilidad y el rojo intenso para representar una máxima vulnerabilidad.

\section{RESULTADOS}

Los factores estresantes naturales (azul) y antropogénicos (rojo) del AMP Uramba obtenidos a través de la revisión de la literatura, los mapas parlantes y los recorridos en barco alrededor del lugar se presentan en la Figura 2. Se identificaron 17 amenazas, de las cuales siete eran naturales y diez antropogénicas. Las más graves y comunes fueron la bioerosión, la erosión hídrica, las aguas residuales, el tráfico marítimo, la contaminación por desechos sólidos y las construcciones humanas. Las localidades más afectadas por las amenazas fueron Ladrilleros (localidad 2), Juanchaco (localidad 5) y Base Naval (localidad 7), ubicadas en la región exterior del AMP, mientras que las localidades menos afectadas se encontraron en la región interior. Los servicios ecosistémicos del AMP Uramba seleccionados de acuerdo con Liquete et al. (2013) se presentan en la Tabla 1. La vulnerabilidad de los valores de los servicios ecosistémicos high, and minimum (0.2) when the probability was very low.

Vulnerability was determinate as a mathematical function of the stressor's magnitude, multiplied by the risk (probability of occurrence and its consequences), and divided by the resilience (capacity of ecosystem recovery) (Kaly et al., 1999; Gowrie, 2003; Turner II et al., 2003; Zacharias and Gregr, 2005).

Ecosystem Service's $=\frac{\text { Ecosystem Service's Stressor } \times \text { Risk }}{}$ Vulnerability

Resilience

For example, for the mudflat ecosystem, we would calculate vulnerability of food provision as follows, with the threat "sea level rise" as a stressor with the maximum value of 3 , risk with the maximum value of 1 , and resilience with the minimum value of 0.2 .

Food provision vulnerability $=\frac{3 \times 1}{0.2}=15$

Due to the overlap in the ranges of values obtained, modulated mainly by the magnitude of the stressor, a color model was generated to facilitate its interpretation using green to represent minimum vulnerability and intense red to represent maximum vulnerability.

\section{RESULTS}

The Uramba MPA natural and anthropogenic stressors identified by the literature review, the story map and the boat field trips around Uramba MPA are presented in blue and red respectively in Figure 2. 17 stressors were identified, from which seven were natural and ten anthropogenic. The most severe and common were bioerosion, water erosion, sewage, marine traffic, solid waste pollution and human constructions. The locations more affected by stressors were Ladrilleros (location 2), Juanchaco (location 5), and Base Naval (location 7), positioned at the outer region of the MPA, while the lesser affected locations were found in the inner region. The ecosystem services selected in Uramba MPA and their descriptions according Liquete et al. (2013) are presented in Table 1. The vulnerability of Uramba MPA ecosystem services values determined by the researchers and professors with a wide knowledge of Uramba MPA are showed in Table 2, they are classifying by ecosystem, ecosystem services and stressors. 


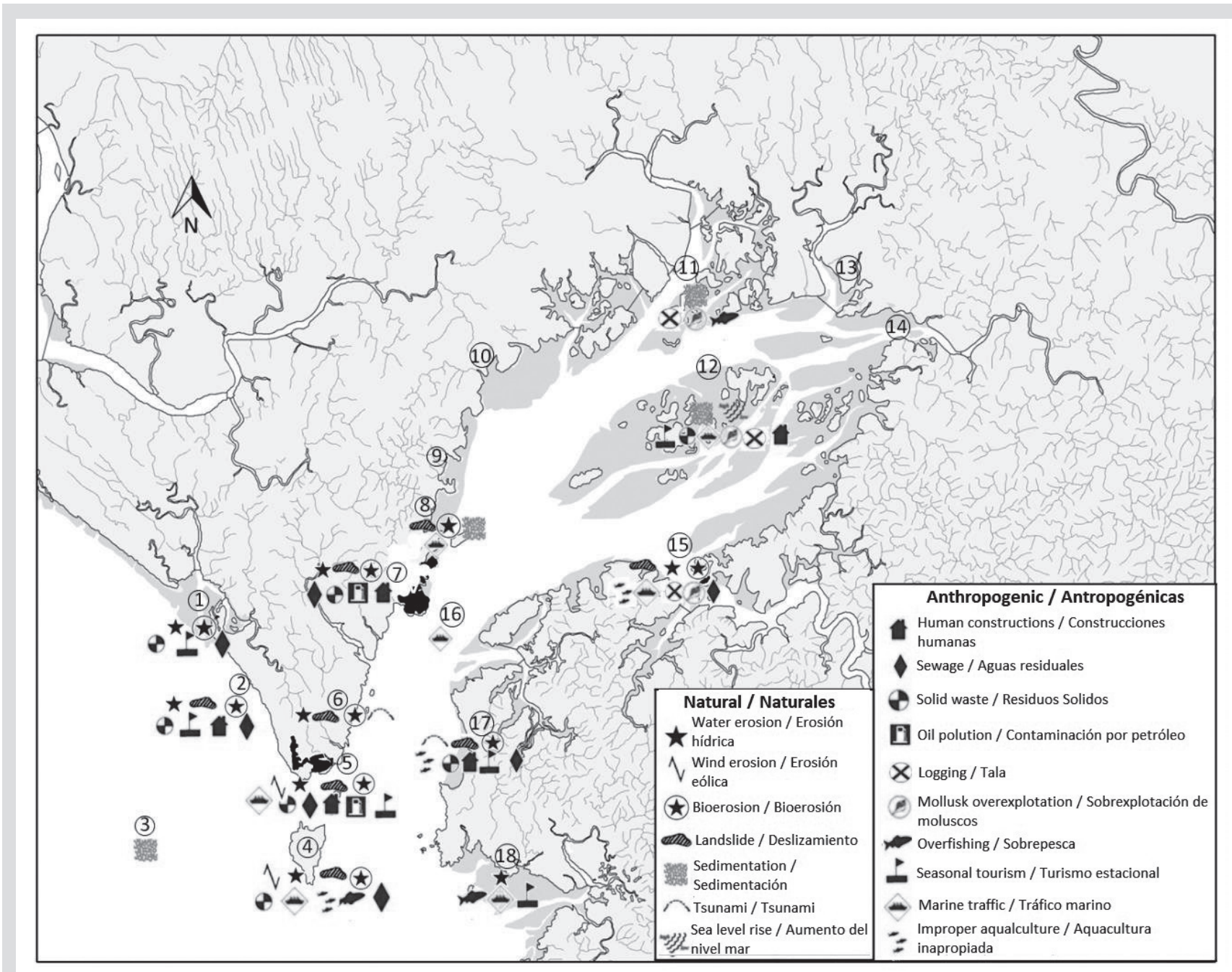

Figura 2. Amenazas naturales y antropogénicas en el AMP Uramba.

Figure 2. Natural and anthropogenic stressors in Uramba MPA.

Tabla 1. Servicios ecosistémicos seleccionados y sus descripciones según clasificación de Liquete et al. (2013).
Table 1. Ecosystem services selected and their descriptions according Liquete et al. (2013) classification.

\begin{tabular}{|l|l|}
\hline \multicolumn{1}{|c|}{ Servicio del ecosistema/Ecosystem Service } & \multicolumn{1}{c}{ Descripción/Description } \\
\hline $\begin{array}{l}\text { Regulación climática/ } \\
\text { Climate regulation }\end{array}$ & $\begin{array}{l}\text { Regulación de gases de efecto invernadero y climáticamente activos/ } \\
\text { Regulation of greenhouse and climate active gases }\end{array}$ \\
$\begin{array}{l}\text { Mantenimiento del ciclo de vida/ } \\
\text { Life cycle maintenance }\end{array}$ & $\begin{array}{l}\text { Sostenimiento que permita la reproducción de especies/ } \\
\text { Support to facilitate species reproduction }\end{array}$ \\
$\begin{array}{l}\text { Suministro de alimentos/Food provision } \\
\text { Protección costera/Coastal protection }\end{array}$ & $\begin{array}{l}\text { Suministro de biomasa para consumo humano/Provision of biomass for human consumption } \\
\text { Protección contra erosión, inundaciones, sequías y eventos extremos/ }\end{array}$ \\
$\begin{array}{l}\text { Recreación y turismo/Recreation and tourism } \\
\text { Valores simbólicos y estéticos/ } \\
\text { Symbolic and aesthetic values }\end{array}$ & $\begin{array}{l}\text { Oportunidades de relajación y diversión/Opportunities for relaxation and amusement } \\
\text { Exaltación de sentidos, emociones o religiones/ } \\
\text { Exaltation of senses, emotions or religions }\end{array}$ \\
\hline
\end{tabular}


del AMP Uramba determinados por los investigadores y profesores expertos en el conocimiento de esta zona se muestran en la Tabla 2. La vulnerabilidad se muestra en términos de los ecosistemas, los servicios ecosistémicos y las amenazas.

Tabla 2. Vulnerabilidad de SE en el AMP Uramba.

Table 2. Vulnerability of ES in Uramba MPA.

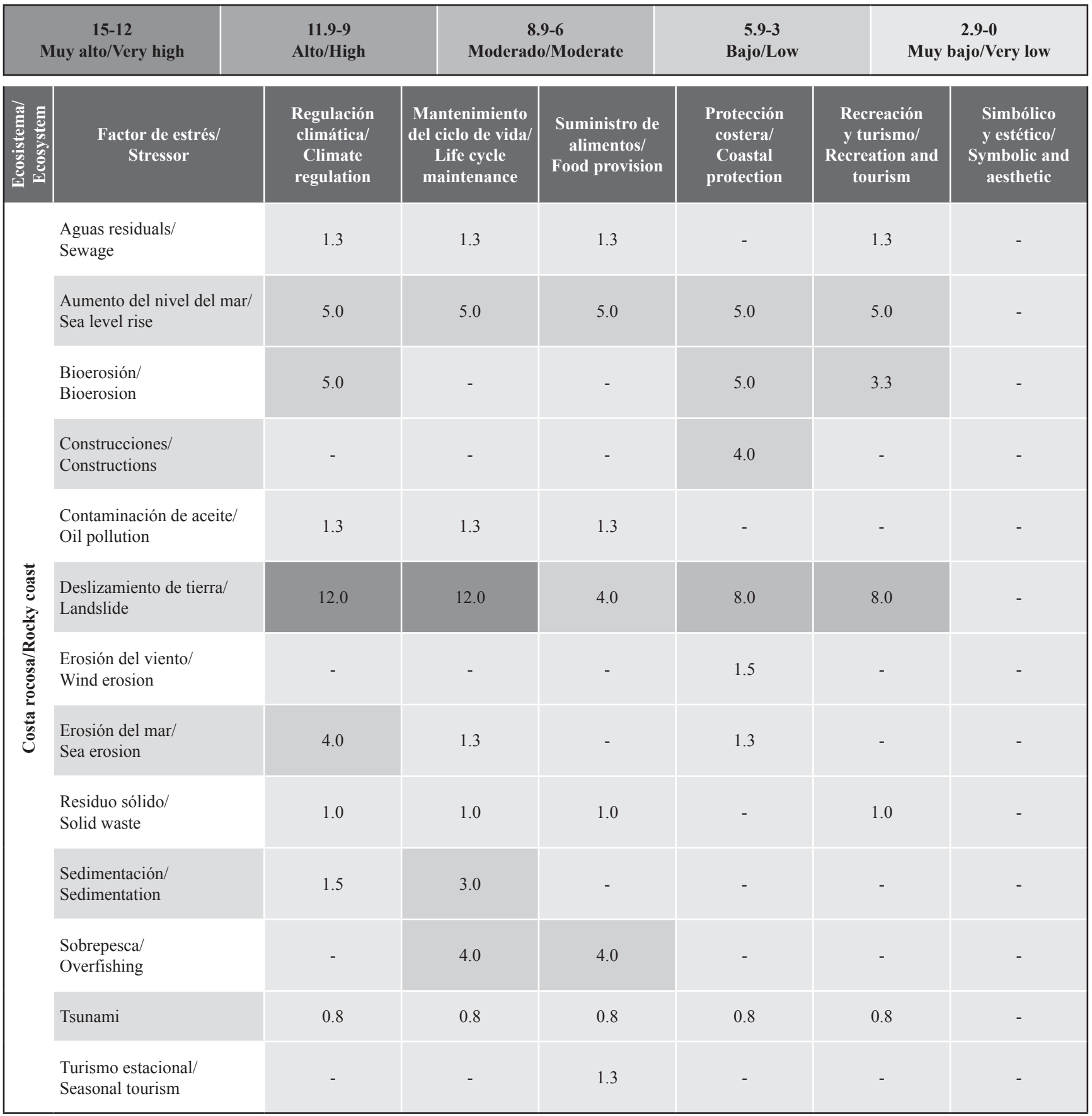




\begin{tabular}{|c|c|c|c|c|c|c|c|}
\hline \multicolumn{2}{|c|}{$\begin{array}{c}15-12 \\
\text { Muy alto/Very high }\end{array}$} & $\begin{array}{c}\text { 11.9-9 } \\
\text { Alto/High }\end{array}$ & \multicolumn{2}{|c|}{$\begin{array}{c}\text { 8.9-6 } \\
\text { Moderado/Moderate }\end{array}$} & $\begin{array}{c}5.9-3 \\
\text { Bajo/Low }\end{array}$ & \multicolumn{2}{|c|}{$\begin{array}{c}2.9-0 \\
\text { Muy bajo/Very low }\end{array}$} \\
\hline 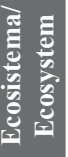 & $\begin{array}{l}\text { Factor de estrés/ } \\
\text { Stressor }\end{array}$ & $\begin{array}{l}\text { Regulación } \\
\text { climática/ } \\
\text { Climate } \\
\text { regulation }\end{array}$ & $\begin{array}{l}\text { Mantenimiento } \\
\text { del ciclo de vida/ } \\
\text { Life cycle } \\
\text { maintenance }\end{array}$ & $\begin{array}{l}\text { Suministro de } \\
\text { alimentos/ } \\
\text { Food provision }\end{array}$ & $\begin{array}{l}\text { Protección } \\
\text { costera/ } \\
\text { Coastal } \\
\text { protection }\end{array}$ & $\begin{array}{c}\text { Recreación } \\
\text { y turismo/ } \\
\text { Recreation and } \\
\text { tourism }\end{array}$ & $\begin{array}{c}\text { Simbólico } \\
\text { y estético/ } \\
\text { Symbolic and } \\
\text { aesthetic }\end{array}$ \\
\hline \multirow{8}{*}{ 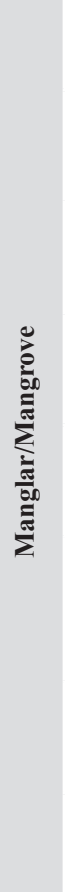 } & $\begin{array}{l}\text { Aguas residuals/ } \\
\text { Sewage }\end{array}$ & 0.5 & 0.5 & 0.5 & - & 0.5 & 0.3 \\
\hline & $\begin{array}{l}\text { Aumento del nivel del mar/ } \\
\text { Sea level rise }\end{array}$ & - & 7.5 & - & 7.5 & 7.5 & - \\
\hline & $\begin{array}{l}\text { Contaminación de aceite/ } \\
\text { Oil pollution }\end{array}$ & 0.7 & 1.3 & 0.7 & - & 0.7 & 0.5 \\
\hline & $\begin{array}{l}\text { Acuicultura/ } \\
\text { Aquaculture }\end{array}$ & - & 0.7 & - & - & - & 0.5 \\
\hline & $\begin{array}{l}\text { Residuo sólido/ } \\
\text { Solid waste }\end{array}$ & 0.8 & 0.8 & - & - & 0.8 & 0.7 \\
\hline & $\begin{array}{l}\text { Sobreexplotación de } \\
\text { moluscos/Mollusk } \\
\text { overexploitation }\end{array}$ & - & 2.7 & 2.7 & - & - & 2.7 \\
\hline & $\begin{array}{l}\text { Sobrepesca/ } \\
\text { Overfishing }\end{array}$ & - & 4.0 & 4.0 & - & - & 4.0 \\
\hline & $\begin{array}{l}\text { Explotación forestal/ } \\
\text { Logging }\end{array}$ & 8.0 & 8.0 & 4.0 & 4.0 & 4.0 & 4.0 \\
\hline \multirow{8}{*}{ 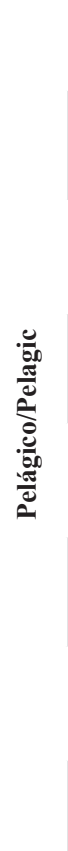 } & $\begin{array}{l}\text { Aguas residuales/ } \\
\text { Sewage }\end{array}$ & 1.3 & - & 1.3 & - & 1.3 & 1.3 \\
\hline & $\begin{array}{l}\text { Contaminación de aceite/ } \\
\text { Oil pollution }\end{array}$ & 1.3 & - & 1.3 & - & 1.3 & 1.3 \\
\hline & $\begin{array}{l}\text { Acuicultura/ } \\
\text { Aquaculture }\end{array}$ & 0.7 & - & - & - & - & - \\
\hline & $\begin{array}{l}\text { Residuo sólido/ } \\
\text { Solid waste }\end{array}$ & 1.3 & - & 1.3 & - & 1.3 & 2.0 \\
\hline & $\begin{array}{l}\text { Sedimentación/ } \\
\text { Sedimentation }\end{array}$ & 2.7 & - & - & - & - & 1.0 \\
\hline & $\begin{array}{l}\text { Sobrepesca/ } \\
\text { Overfishing }\end{array}$ & - & - & 8.0 & - & - & - \\
\hline & $\begin{array}{l}\text { Tráfico marítimo/ } \\
\text { Marine traffic }\end{array}$ & - & - & - & - & 2.0 & - \\
\hline & $\begin{array}{l}\text { Turismo estacional/ } \\
\text { Seasonal tourism }\end{array}$ & - & - & 1.3 & - & - & - \\
\hline
\end{tabular}




\begin{tabular}{|c|c|c|c|c|c|c|c|}
\hline \multicolumn{2}{|c|}{$\begin{array}{c}15-12 \\
\text { Muy alto/Very high }\end{array}$} & $\begin{array}{l}\text { 11.9-9 } \\
\text { Alto/High }\end{array}$ & \multicolumn{2}{|c|}{$\begin{array}{c}\text { 8.9-6 } \\
\text { Moderado/Moderate }\end{array}$} & $\begin{array}{c}\text { 5.9-3 } \\
\text { Bajo/Lov }\end{array}$ & \multicolumn{2}{|c|}{$\begin{array}{c}2.9-0 \\
\text { Muy bajo/Very low }\end{array}$} \\
\hline 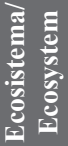 & $\begin{array}{l}\text { Factor de estrés/ } \\
\text { Stressor }\end{array}$ & $\begin{array}{l}\text { Regulación } \\
\text { climática/ } \\
\text { Climate } \\
\text { regulation }\end{array}$ & $\begin{array}{l}\text { Mantenimiento } \\
\text { del ciclo de vida/ } \\
\text { Life cycle } \\
\text { maintenance }\end{array}$ & $\begin{array}{l}\text { Suministro de } \\
\text { alimentos/ } \\
\text { Food provision }\end{array}$ & $\begin{array}{l}\text { Protección } \\
\text { costera/ } \\
\text { Coastal } \\
\text { protection }\end{array}$ & $\begin{array}{c}\text { Recreación } \\
\text { y turismo/ } \\
\text { Recreation and } \\
\text { tourism }\end{array}$ & $\begin{array}{l}\text { Simbólico } \\
\text { y estético/ } \\
\text { Symbolic and } \\
\text { aesthetic }\end{array}$ \\
\hline \multirow{6}{*}{ 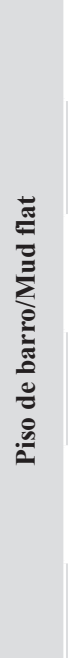 } & $\begin{array}{l}\text { Aguas residuales/ } \\
\text { Sewage }\end{array}$ & 0.5 & 0.5 & 0.5 & - & - & - \\
\hline & $\begin{array}{l}\text { Sea level rise/ } \\
\text { Aumento del nivel del mar }\end{array}$ & 15.0 & - & 15.0 & 15.0 & - & - \\
\hline & $\begin{array}{l}\text { Contaminación de aceite/ } \\
\text { Oil pollution }\end{array}$ & 1.0 & 1.0 & 1.0 & - & - & - \\
\hline & $\begin{array}{l}\text { Residuo sólido/ } \\
\text { Solid waste }\end{array}$ & 0.7 & 0.7 & - & - & - & - \\
\hline & $\begin{array}{l}\text { Sedimentación/ } \\
\text { Sedimentation }\end{array}$ & 2.5 & - & - & - & - & - \\
\hline & $\begin{array}{l}\text { Sobrepesca/ } \\
\text { Overfishing }\end{array}$ & - & 1.0 & 1.0 & - & - & - \\
\hline \multirow{7}{*}{ 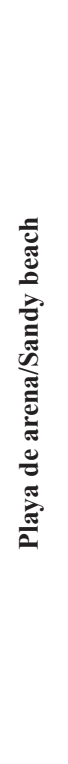 } & $\begin{array}{l}\text { Aguas residuales/ } \\
\text { Sewage }\end{array}$ & 1.3 & 1.3 & - & - & 1.3 & 1.3 \\
\hline & $\begin{array}{l}\text { Aumento del nivel del mar/ } \\
\text { Sea level rise }\end{array}$ & 15.0 & - & - & 15.0 & 15.0 & - \\
\hline & $\begin{array}{l}\text { Construcciones/ } \\
\text { Constructions }\end{array}$ & - & - & - & - & 1.3 & - \\
\hline & $\begin{array}{l}\text { Contaminación de aceite/ } \\
\text { Oil pollution }\end{array}$ & 2.0 & 2.0 & - & - & 2.0 & 2.0 \\
\hline & $\begin{array}{l}\text { Residuo sólido/ } \\
\text { Solid waste }\end{array}$ & 1.7 & 1.7 & - & - & 1.7 & 1.7 \\
\hline & $\begin{array}{l}\text { Sedimentación/ } \\
\text { Sedimentation }\end{array}$ & 5.0 & 5.0 & - & - & - & - \\
\hline & $\begin{array}{l}\text { Turismo estacional/ } \\
\text { Seasonal tourism }\end{array}$ & - & 1.3 & - & - & - & - \\
\hline
\end{tabular}




\section{DISCUSIÓN}

El AMP Uramba contiene todos los ecosistemas costeros y marinos que se encuentran a lo largo del Pacífico colombiano, excepto los arrecifes de coral, aunque existen registros de colonias de coral aisladas (Pocillopora damicornis y $P$. capitata) en Los Negritos (localidad 3, Figura 1). Las playas de arena se encuentran en los dos límites exteriores del AMP Uramba, al norte de La Barra (localidad 1, Figura 1), Ladrilleros (localidad 2, Figura 1), Juanchaco (localidad 5, Figura 1) y al sur de Chucheros (localidad 17, Figura 1) y El Tigre (ubicación 18, Figura 1). Estas playas están formadas por la acumulación de arena transportada por ríos y procesos hidrodinámicos marinos, enriquecida con materia orgánica derivada de la descomposición de los restos vegetales de los manglares. Estas playas presentan un uso estacional intensivo por actividades turísticas, por lo cual están contaminadas por residuos sólidos, principalmente plásticos (Riascos et al., 2019). La costa rocosa, que contiene acantilados $\mathrm{y}$ playas rocosas derivadas de la erosión de estos acantilados, es el ecosistema más abundante del AMP Uramba. Algunas de estas formaciones rocosas constituyen islas o islotes, incluyendo una formación rocosa aislada fuera del AMP llamada Los Negritos (localidad 3, Figura 1). En la región interior de La Plata existen importantes arrecifes rocosos (localidad 1, 12). Estos se encuentran en activo proceso de erosión debido al oleaje y la acción intensa de organismos bioerosionadores (Cantera et al., 1998; Cobo-Viveros y Cantera, 2015; Cantera, 2016). La bahía presenta importantes parches de manglares en buen estado de conservación en la región norte, así como extensas áreas en el interior y la región sur, con cerca de 3000 ha conservadas en las cuales habitan las especies Rhizophora mangle, R. racemosa, Avicennia germinans, Laguncularia racemosa, Conocarpus erectus, Pelliciera rhizophorae y Mora oleifera. Estos manglares no son tan complejos como los del sur de Colombia. Sin embargo, pueden alcanzar los $35 \mathrm{~m}$ en la región interior del AMP Uramba en Quebrada Valencia (localidad 11, Figura 1), Agujeros (localidad 13, Figura 1), Luisico (localidad 14, Figura 1) y La Sierpe (localidad 15, Figura 1). Los manglares del AMP Uramba y sus recursos asociados (madera, peces, crustáceos, moluscos y vida silvestre) están sujetos a explotación artesanal por parte de los habitantes de la región. Los hábitats pelágicos están en relativa buena condición, ya que los niveles de contaminación son bajos.

Las amenazas naturales y antropogénicas en el AMP Uramba afectan principalmente la región externa de la bahía Ladrilleros (localidad 2), Juanchaco (localidad

\section{DISCUSSION}

Uramba MPA contains all the main coastal and marine ecosystems found along the Colombian Pacific coast with the exception of coral reefs, although there are records of isolated coral colonies (Pocillopora damicornis and $P$. capitata) in Los Negritos (location 3, Figure 1). Sandy beaches are found along the two outer limits of the Uramba MPA, at north La Barra (location 1, Figure 1), Ladrilleros (location 2, Figure 1), Juanchaco (location 5, Figure 1), and at south Chucheros (location 17, Figure 1), and El Tigre (location 18, Figure 1). These beaches are formed by the accumulation of sand carried by rivers and marine hydrodynamic processes, are enriched by organic matter derived from the decomposition of plant debris from the mangroves and, because of their intensive seasonal use in tourism activities, are contaminated in a significant way by solid waste, mainly plastic (Riascos et al., 2019). The rocky coast, containing cliffs and rocky beaches derived from the erosion of these cliffs, is the most abundant ecosystem. Some of these rocky formations form islands or islets, and even an isolated rocky formation outside the Uramba MPA called Los Negritos (location 3, Figure 1). There are important intertidal offshore rocky reefs in the inner part of La Plata (location 12, Figure 1), which are rocky formations composed of about 10 small rocky islands and islets. These rocky formations are in the active process of erosion due to seasonally strong waves in some regions and facilitated by organisms in an intense process of bioerosion (Cantera et al., 1998; Cobo-Viveros and Cantera, 2015; Cantera, 2016). The bay presents important patches of mangroves in a good state of conservation in the north region and extensive areas in the interior and south border, with about 3000 ha conserved (Rhizophora mangle, R. racemosa, Avicennia germinans, Laguncularia racemosa, Conocarpus erectus, Pelliciera rhizophorae and Mora oleifera). These mangroves are not as complex as the mangroves of the south of Colombia. However, mangroves can reach $35 \mathrm{~m}$ in the interior region of Uramba MPA in Quebrada Valencia (location 11, Figure 1), Agujeros (location 13, Figure 1), Luisico (location 14, Figure 1), and La Sierpe (location 15, Figure 1). All the mangroves and their biodiversity (wood, fish, crustaceans, mollusks and wildlife) are subject to artisanal exploitation by the inhabitants of the region. The pelagic habitats are in good condition since the contamination levels are low.

The assessment of Uramba MPA identified numerous natural and anthropogenic potential stressors 
5) y Base Naval (localidad 7). Las principales amenazas naturales encontradas fueron la erosión hídrica, la bioerosión y los deslizamientos de tierra. Es importante aclarar que es poco lo que se puede hacer para manejar estas amenazas; sin embargo, se pueden adelantar acciones para mitigar el riesgo que estas representan para los asentamientos humanos en el AMP Uramba. Los efectos sinérgicos causados por la combinación de erosión hídrica, bioerosión y deslizamientos de tierra tienden a ser más intensos en la región externa de las bahías, ya que estas zonas están más expuestas al efecto de olas, vientos y tormentas (Cantera, 1991; Posada et al., 2009). Una gran cantidad de amenazas antropogénicas puede estar asociada a los asentamientos humanos, los cuales pueden sumar hasta 50000 personas en temporada alta de turismo y 30000 residentes (DANE, 2012). Las amenazas asociadas con los asentamientos humanos y sus actividades son las aguas residuales, los desechos sólidos, las construcciones y la contaminación por petróleo. En el AMP Uramba, la eliminación de aguas residuales y desechos sólidos no se realiza adecuadamente. Las construcciones humanas contribuyen a la pérdida de hábitat natural. Los derrames de hidrocarburos se generan accidental o arbitrariamente durante las operaciones de navegación. La región interna del AMP Uramba presenta menos amenazas, probablemente debido al tamaño de las poblaciones que en ella habitan (i.e. La Plata, localidad 12, con aproximadamente 200 personas [DANE, 2012]). Además, le región interna se encuentra más protegida de fenómenos naturales como olas, vientos y tormentas (Cantera, 1991).

Las amenazas antropogénicas y naturales pueden transformar, degradar o limitar la prestación de servicios ecosistémicos. Los servicios de los ecosistemas marinos se han investigado ampliamente, con énfasis en los manglares (Duke et al., 2007; Barbier et al., 2011; Vo et al., 2012; Liquete et al., 2013; Mukherjee et al., 2014; Thompson et al., 2019), corales (Moberg, et al., 1999; Laurans et al., 2013; Woodhead et al., 2019) y costas rocosas (Wilson, 2005; Branch et al., 2008; Marcelli et al., 2018). En Colombia, se han realizado investigaciones enfocadas en los servicios de los ecosistemas marinos tanto en el Caribe (Blanco et al., 2012; Aldana-Domínguez et al., 2017) como en las costas del Pacífico de Colombia (Cantera et al., 2013; Palacios y Cantera, 2017; Mejía Rentería et al., 2019; Palacios et al., 2019; Rojas et al., 2019). Cantera et al. (2013) identificaron los ecosistemas de manglares y costas rocosas como sistemas clave para los asentamientos humanos en el AMP Uramba, debido a su alta provisión de servicios ecosistémicos. Palacios affecting principally the outer locations Ladrilleros (location 2), Juanchaco (location 5), and Base Naval (location 7). The main natural stressors found at these locations were water erosion, bioerosion and landslides. It is important to clarify that is less that can be done to manage tis stressors, however actions can be conducted to mitigate the risk that they represent to human settlements in Uramba MPA. The synergic effects caused by the combination of water erosion, bioerosion and landslides tend to be more intense in the outer locations as they are more exposed to waves, winds, and storms (Cantera, 1991; Posada et al., 2009). The great number of anthropogenic stressors in these locations could be explained by the human settlements with a population size of approximately 30000 people, a number that reaches up to 50000 people in the tourismrelated high season (DANE, 2012). Stressors associated with human settlements and their activities are sewage, solid waste, human constructions and oil pollution at these locations. Sewage and waste disposal are not properly managed in the area. Human constructions may enhance natural habitat loss. Oil spills generated accidentally or arbitrarily during boating operations stress the marine and coastal systems of the MPA. On the contrary, the inner region of the Uramba MPA presents fewer stressors. This can probably be accounted for by the low population living there (i.e. La Plata, location 12, with approximately 200 people [DANE, 2012]), and the fact that its ecosystems are less exposed to natural phenomena such as waves, winds, and storms (Cantera, 1991).

Anthropogenic and natural stressors can transform, degrade, or limit the provision of Ecosystem Services. Marine ecosystems services have been researched widely, with research focused on mangroves (Duke et al., 2007; Barbier et al., 2011; Vo et al., 2012; Liquete et al., 2013; Mukherjee et al., 2014; Thompson et al., 2019), corals (Moberg et al., 1999; Laurans et al., 2013; Woodhead et al., 2019) and rocky coasts (Wilson, 2005; Branch et al., 2008; Marcelli et al., 2018). In Colombia, research has been conducted on marine ecosystem services on both Caribbean (Blanco et al., 2012; Aldana-Domínguez et al., 2017) and Pacific coasts of Colombia (Cantera et al., 2013; Palacios and Cantera, 2017; Mejía Rentería et al., 2019; Palacios et al., 2019; Rojas et al., 2019). Cantera et al. (2013) identified mangrove and rocky coast ecosystems as key systems for the human settlements in Uramba MPA. Palacios and Cantera (2017) and Palacios et al. (2019) identified food provision as a key ecosystem 
y Cantera (2017) y Palacios et al. (2019) identificaron la provisión de alimentos como un servicio ecosistémico clave brindado por el AMP Uramba. Los manglares, planos lodosos y arrecifes rocosos se han identificado como áreas de sala-cuna en las cuales larvas y juveniles de especies de importancia económica y ecológica encuentran alimento y protección (Invemar-Univalle, 2011). El AMP Uramba está protegida como Parque Nacional Natural; por tanto, es importante conservar los servicios ecosistémicos, así como su integridad espacial y temporal (Cantera et al., 2013).

Las planos lodosos y playas arenosas del AMP Uramba se caracterizan por tener una pendiente muy baja, lo que expresa su vulnerabilidad en un escenario de aumento del nivel del mar (Olivo et al., 2011). Para superar dicho aumento, estos ecosistemas deben tener una tasa de acreción más alta que la tasa de aumento del nivel del mar. De lo contrario, las inundaciones serán inevitables y ocasionarán una mayor erosión. La inundación causada por el aumento del nivel del mar y los cambios en el ciclo de acreción-erosión afectarán la actividad microbiana (Chambers et al., 2013) y provocarán posibles alteraciones en los ciclos de carbono, fósforo y nitrógeno (Steinmuller y Chambers, 2019). Como resultado, la regulación climática se verá afectada por el aumento en el nivel del mar de las playas arenosas y planos lodosos del AMP Uramba. Los cambios en la regulación climática podrían acelerar las tasas de mineralización o lixiviación en los suelos de los humedales costeros y producir floraciones de algas nocivas y eutrofización (Steinmuller y Chambers, 2019).

Los planos lodosos y las playas de arena sirven como zonas de amortiguamiento naturales que protegen la costa y los ecosistemas costeros de las olas, las marejadas, las mareas y las inundaciones marinas. La vulnerabilidad de las playas arenosas y los planos lodosos frente al aumento en el nivel del mar ha sido identificado globalmente por otros estudios (Simm, 1996; Doody, 2012; Dominati, 2014; IPCC, 2019). Este aumento reduce la capacidad de estos sistemas para proteger la costa ante erosión, tormentas y tsunamis (Hanley et al., 2014).

Los organismos que habitan en los planos lodosos requieren salinidades relativamente bajas y temperaturas altas. El aumento en el nivel del mar y la inundación asociada elevarán la salinidad, disminuirán la temperatura y cambiarán los patrones de circulación de estos sistemas. Como respuesta, la estructura de la comunidad y las redes tróficas cambiarán. Por lo tanto, su provisión de alimentos podría verse modificada $\mathrm{y} / \mathrm{o}$ afectada. service provided by mangroves in the Colombian Pacific and in Uramba MPA. It has been demonstrated that the Bay contributes to climate regulation throughout southwestern Colombia and to the functioning of mangroves, mud flats and rocky reefs as nurseries in which larvae and juvenile forms of many economically and ecologically important species find food and protection (Invemar-Univalle, 2011). The entire bay area is protected as a National Natural Park, which implies that these ecosystem services retain their spatial and temporal integrity (Cantera et al., 2013).

Uramba MPA mud flats and sandy beaches are characterized by a very low slope, indicating their vulnerability in a sea level rise scenario (Olivo et al., 2011). To overcome sea level rise, they must accrete faster than the sea level rises. Otherwise, flooding is inevitable, and it will be followed by increased erosion. The inundation caused by sea level rise and the changes to the accretion-erosion cycle will affect microbial activity (Chambers et al., 2013). It will cause alterations to the rate of carbon sequestration, carbon balance, and phosphorus and nitrogen exportation (Steinmuller and Chambers, 2019). As a result, climate regulation will be affected in Uramba MPA sandy beaches and mud flats. The changes of climate regulation could accelerate mineralization rates or leaching in coastal wetland soils, and it may produce harmful algal blooms and eutrophication (Steinmuller and Chambers, 2019).

Mud flats and sandy beaches are widely known to provide coastal protection. These systems serve as natural buffer zones that protect the coastline and back shore coastal ecosystems from waves, surges, tides, and marine flooding. The vulnerability of sandy beaches and mud flats to sea level rise has been discussed globally by other studies (Simm, 1996; Doody, 2012; Dominati, 2014; IPCC, 2019). Sea level rise reduces their capability to protect the coast against storms, high waves such as tsunamis, and ecosystem erosion (Hanley et al., 2014).

Organisms that live in mud flats have environmental requirements related to relatively low salinity and high temperature. Sea level rise and its associated inundation will raise the salinity, lower the temperature, and change the circulation patterns of these systems. As an answer, the community structure and the food webs will change. Hence, the mud flat ES of food provision could be modified and/or affected. 
Las playas de arena son universalmente reconocidas por su valor recreativo y por producir altos ingresos económicos asociados con el turismo costero (King y Symes, 2004). Las playas son valoradas por residentes y turistas gracias a su valor cultural, estético y recreativo (Defeo et al., 2009). No obstante, el aumento en el nivel del mar podría ocasionar tanto pérdida de hábitat de playa a causa de la migración y la erosión de la costa, como una menor deposición de arena nueva a través del transporte litoral (King et al., 2018). En el AMP Uramba, la pérdida del SE de recreación y turismo afectaría las actividades económicas en La Barra (localidad 1), Ladrilleros (localidad 2) y Juanchaco (localidad 5). Las playas en estas localidades son reconocidas como importantes destinos turísticos en la costa del Pacífico colombiano. La economía local depende en gran medida del turismo. Los residentes brindan servicios de alojamiento, comida, instalaciones comerciales y visitas guiadas a paisajes naturales cercanos (Cantera et al., 2013).

La costa rocosa ubicada en la región externa del AMP Uramba está expuesta a la fuerte acción de las olas y sometida a una fuerte erosión. La erosión causada por la fuerza de las olas se intensifica por las fuerzas del viento y el efecto de los organismos bioerosionadores, que perforan el sustrato rocoso en busca de las algas que crecen sobre este. La bioerosión y la erosión hídrica son las principales amenazas naturales identificadas en el AMP Uramba. Estas han sido documentadas como principales causas de impacto en el AMP Uramba (Cantera, 1991; Cobo-Viveros, 2007; Posada et al., 2009; Cantera et al., 2013). Los efectos sinérgicos de los procesos de erosión aceleran la caída, la descomposición y la erosión de acantilados y playas rocosas, provocando deslizamientos. En el AMP Uramba, estos representan el mayor riesgo para el SE del suministro de alimentos. Los deslizamientos tienen efectos sobre la regulación climática, la protección costera y la recreación y el turismo. La erosión de la costa rocosa y los deslizamientos de tierra del AMP Uramba podrían vulnerar el SE de protección costera. La erosión costera, en combinación con las construcciones humanas, viola la integridad estructural de la costa rocosa y su capacidad para brindar protección costera.

Las costas rocosas dispersan sedimentos al litoral y al mar abierto cuando sufren procesos erosivos. Si aumenta la cantidad de material dispersado, las propiedades físicas y químicas de los sistemas circundantes se verán afectadas (Violante, 2009). En el escenario de aumento en el nivel del mar, los cambios asociados al desgaste químico de la costa rocosa pueden tener efectos sobre el SE de regulación climática.
Sandy beaches are universally recognized for their important recreational value, and producing high economic revenue associated with coastal tourism worldwide (King and Symes, 2004). Beaches are appraised by residents and tourists for their cultural, aesthetic, and recreational value (Defeo et al., 2009). Increased loss of beach area can be associated with sea level rise, with subsequent losses of beach habitat caused by shoreline migration and erosion, and reduced deposition of new sand via littoral transport (King et al., 2018). In Uramba MPA, loss of its recreation and tourism ES will affect economic activities in Ladrilleros (location 2) and Juanchaco (location 5). Their beaches are well recognized as tourist destinations in the Colombian Pacific coast. The local economy depends heavily on tourism. Residents provide accommodation services, food, commercial facilities, and guided tours to nearby natural landscapes (Cantera et al., 2013).

The rocky coast located at the outer region of Uramba MPA is exposed to strong wave action and subjected to heavy erosion. Erosion caused by wave forces is further reinforced by wind forces and bioeroders that graze algae and bore the substrate. Bioerosion and water erosion were the main natural stressors identified in Uramba MPA by the present study. These stressors have been documented as major causes of impact in the area (Cantera, 1991; Cobo-Viveros, 2007; Posada et al., 2009; Cantera et al., 2013). These combined effects of erosion processes accelerate the fall, decomposition, and weathering of cliffs and rocky beaches, causing landslides. In Uramba MPA's rocky coast, landslides represent the highest risk for the ES of food provision. Landslides harm climate regulation, coastal protection, and recreation and tourism. Uramba MPA's rocky coast erosion and landslides lead to the loss of their capacity to provide coastal protection. Coastal erosion in combination with human construction threaten the structural integrity of the rocky coast and its ability to provide coastal protection.

Rocky coasts deliver sediments to the littoral and the open sea through erosion. If the amount of the output material increases, the physical and chemical properties of the surrounding systems will be affected (Violante, 2009). Chemical weathering may have an important effect through the movement of major masses, such as in a landslide scenario, and these changes will modify climate regulation in Uramba MPA's rocky coasts.

Landslides and erosion can change the habitats that serve to maintain life cycles, especially tidal pools. 
Los deslizamientos y la erosión pueden vulnerar el SE de mantenimiento de los ciclos de vida, especialmente en los charcos rocosos intermareales. Estos han sido identificados como zonas importantes de sala-cunas, en donde una gran cantidad de organismos habita durante sus primeras etapas ontogenéticas, ya que sus condiciones permiten el crecimiento y, además, brindan refugio ante depredadores (Dias et al., 2016). Los cambios estructurales y la pérdida de charcos rocosos intermareales pueden vulnerar la capacidad de la costa rocosa del AMP Uramba para mantener los ciclos de reproducción.

Otros ecosistemas afectados por el aumento del nivel del mar son los manglares. Aunque estos se encuentran en condiciones de cambio constante, la estratificación de las diferentes especies de manglares está estrechamente relacionada con los cambios de marea. En dicha estratificación, las especies mejor adaptadas a altos niveles de salinidad se ubican en la parte baja de la línea de marea mientras que las especies con menos tolerancia se ubican en la parte alta. Debido a los procesos de erosión y sedimentación, el ecosistema está en constante migración. Si la tasa de aumento del nivel del mar excede la de migración de los manglares, este probablemente se inundará y los arboles morirán. Por el contrario, si el aumento del nivel del mar es relativamente lento, los manglares pueden permanecer en la costa a través de la acumulación de sedimentos verticalmente (YáñezArancibia et al., 1998; Coll et al., 2001). Si los manglares del AMP Uramba no pueden contrarrestar la tasa de aumento del nivel del mar, SE como la protección costera, el mantenimiento del ciclo de vida, la recreación y el turismo están en juego. La tala y destrucción de áreas de manglar conducirá a la pérdida de hábitat muy importante, vulnerando la base de las redes tróficas en el Pacífico colombiano (Univalle, 2010; InvemarUnivalle, 2011).

El SE de mantenimiento de ciclo de vida de los manglares del AMP Uramba ha sido ampliamente estudiado. Estos manglares proporcionan condiciones fisicoquímicas adecuadas, refugio y fuentes de alimento ideal para las primeras etapas de vida de muchas especies marinas y estuarinas. Los manglares del AMP se han identificado como de gran importancia para la reproducción de especies ecológica y comercialmente relevantes en el Océano Pacífico colombiano (Invemar-Univalle-Inciva, 2006; Cantera et al., 2013). El SE de mantenimiento de ciclo de vida en el AMP Uramba se encuentra amenazado por la tala y el aumento del nivel del mar.
The use of tidal pools during early ontogenetic stages is common, as their conditions allow for enhanced growth and provide refuge from larger predators (Dias et al., 2016). Structural changes or loss of rocky tidal pools may undermine the capacity of Uramba MPA's rocky coast to maintain life cycles.

Other ecosystems heavily affected by sea level rise are mangroves. Although mangroves are known to be in a system of constant change, the stratification of the different species of mangroves is closely related to tidal changes, with species better adapted to high levels of salinity in the lower part of the tidal line and species with less tolerance in the upper part. Due to the processes of erosion and sedimentation, the ecosystem is in constant migration with changes in the distribution of these species. If the rate of sea level rise exceeds that of mangrove migration, the ecosystem will sink and probably be replaced. In contrast, if the increase in sea level is relatively slow, mangroves can remain on the shoreline by accumulating sediments vertically and growing on them (Yáñez-Arancibia et al., 1998; Coll et al., 2001). If Uramba MPA mangroves cannot deal with the rate of sea level rise, ES such as coastal protection, life cycle maintenance, and recreation and tourism are at stake. The logging and destruction of mangrove areas will lead to the loss of a very important habitat and the base of the food web in the Colombian Pacific Ocean (Univalle, 2010; Invemar-Univalle, 2011).

Uramba MPA mangroves have been studied extensively due to their characteristics as a key habitat for breeding and larval development. Mangroves provide adequate physicochemical conditions, refuge and food sources for early life stages. Furthermore, they are important to the reproduction of ecologically and commercially important species in the Colombian Pacific Ocean (Invemar-Univalle-Inciva, 2006; Cantera et al., 2013). Such characteristics make mangroves important for life cycle maintenance, an ecosystem service that is threatened by logging and sea level rise in Uramba MPA.

Mangroves provide coastal protection by attenuating waves and surge, dissipating wave energy through their root systems and trunks, reducing windrelated damage, protecting from tsunamis, hurricanes, and storms, stabilizing the soil, and preventing soil salination. As such, mangroves protect human populations from natural disasters (Mazda et al., 2006; Alongi, 2008; Darryl et al., 2015), a feature that is undermined in the face of sea level rise. 
Los manglares proveen SE de protección costera al disipar la energía de las olas a través de sus sistemas de raíces $\mathrm{y}$ troncos, reducir el daño relacionado con el viento, proteger contra tsunamis, huracanes y tormentas, estabilizar el suelo y prevenir la salinización del suelo. Por tanto, los manglares protegen a las poblaciones humanas de los desastres naturales (Mazda et al., 2006; Alongi, 2008; Darryl et al., 2015). Este SE está vulnerado por la probabilidad del aumento en el nivel del mar.

El uso turístico del manglar en el AMP Uramba y en el mundo incluye actividades recreativas como caminatas, paseos en bote, observación de vida silvestre y pesca (Spalding y Parrett, 2019). Este uso de alto valor y bajo impacto de los manglares del AMP Uramba será vulnerable en escenarios de aumento agudo del nivel del mar.

Evaluar los efectos del aumento del nivel del mar es clave para comprender cómo el cambio climático afecta los SE. Dado que la mayoría de los ecosistemas evaluados en el AMP Uramba se encuentran en la interface costeromarina, esto los hace muy vulnerables a la amenaza del aumento en el nivel del mar (Osland et al., 2016). Para ello, tener en cuenta el conocimiento tradicional ecológico de los actores locales puede ser beneficioso para priorizar las acciones de adaptación dirigidas a reducir la vulnerabilidad socioeconómica. (Metcalf et al., 2015). Osland et al. (2016) sugieren incluir factores macroclimáticos como mediciones temporales de temperatura y lluvia en estudios futuros, ya que estos influencian la estructura y función de los ecosistemas.

Otra amenaza presente en el AMP Uramba es la sobrepesca. La pesca se lleva a cabo en toda el AMP y de esta depende gran parte de las poblaciones humanas para su subsistencia. Las actividades pesqueras en el AMP Uramba son prácticas ancestrales de supervivencia, principalmente artesanales. La pesca tiene como finalidad la subsistencia, el abastecimiento del sector turístico y la venta en los mercados de Buenaventura, la principal ciudad más cercana al AMP Uramba. Hay más de 1500 pescadores artesanales en el AMP Uramba, y su captura anual por año es de aproximadamente 170 toneladas de pescado y 200 toneladas de camarón (Cantera et al., 2013). Los pescadores dentro del AMP utilizan artes de pesca no reguladas, pescan en áreas de salacuna y desarrollan intensos esfuerzos. Estas prácticas tienen impactos negativos en los ecosistemas pelágicos del AMP Uramba como el colapso de las poblaciones de peces, la desestabilización del ecosistema dentro y fuera del AMP y los SE de provisión de alimento en las zonas pelágicas (Rubio, 1984; Gislason et al., 2000; Halpern et al., 2008; FAO, 2010).
Another mangrove ES that could be affected is recreation and tourism. Recreational activities include hiking, boating, wildlife-watching, and fishing (Spalding and Parrett, 2019). This high-value and low impact use of Uramba MPA mangroves will be vulnerable in scenarios of acute sea level rise.

Evaluating the effects of sea level rise is key to understanding how climate change affects ES and evaluating its possible impact. Given that most of the ecosystems evaluated in Uramba MPA are found at the coastal-marine interface, it makes them very vulnerable to this threat (Osland et al., 2016). In this sense, taking local stakeholders' knowledge into account can be beneficial for prioritizing adaptation actions to reduce socioeconomic vulnerability (Metcalf et al., 2015). Additionally, Osland et al. (2016) suggest including macroclimatic drivers such as temperature and rainfall regimes in future studies since they have a strong influence on structure and function of the ecosystems.

The last main stressor to the ES is overfishing. This activity is carried out in the entire area on which a large part of the inhabiting population is dependent. Fishing activities in Uramba MPA are ancestral survival practices, and mainly artisanal. The fishing purposes are subsistence, to supply the tourism sector, and to sell in the markets of Buenaventura, the main city near Uramba MPA. There are more than 1500 artisanal fishermen in Uramba MPA, and their annual catch per year is approximately 170 tons of fish and 200 tons of shrimp (Cantera et al., 2013). Fishermen inside the MPA use unregulated fishing gear, fish in areas of breeding, and develop intense fishing efforts. These practices have negative impacts on Uramba MPA pelagic ecosystems, such as the collapse of fish populations, ecosystem destabilization inside and outside the MPA, and threatening ES of food provision in pelagic zones (Rubio, 1984; Gislason et al., 2000; Halpern et al., 2008; FAO, 2010).

Ecosystems services at Uramba MPA present a low ecological resilience when faced with sea level rise and overfishing. Threats like logging, landslides and oil pollution also strongly affect the vulnerability of mangroves, rocky coasts and sandy beaches, respectively. In these cases, ecological resilience is useful because there is significant risk to a persistent shift (Mumby et al., 2014; Carr et al., 2017). Regarding sewage, although it is a recurring stressor and may affect processes that link pelagic and benthonic ecosystems, the high volumes of 
Los SE en el AMP Uramba presentan una baja resiliencia ecológica ante el aumento del nivel del mar y la sobrepesca. Amenazas como la tala, los deslizamientos de tierra y la contaminación del suelo también afectan fuertemente la vulnerabilidad de los manglares, las costas rocosas y las playas de arena. En el caso del AMP, es útil estudiar su resiliencia ecológica debido a que existe un riesgo significativo de cambio persistente (Mumby et al., 2014; Carr et al., 2017). En relación con la amenaza de aguas residuales, a pesar de que este es un factor de estrés recurrente y puede afectar procesos que vinculan ecosistemas pelágicos y bentónicos, los altos volúmenes de agua del océano disminuyen su impacto y la vulnerabilidad a esta amenaza. Se han identificado resultados similares en el mar Báltico (Griffiths et al., 2017), que indican que el sistema en su conjunto exhibe un gran potencial de recuperación, incluso cuando está muy perturbado (Mumby et al., 2014).

\section{CONCLUSIONES}

Se lograron identificar y mapear los SE ofrecidos por los ecosistemas marinos del AMP Uramba, sus amenazas y su vulnerabilidad a través de la recopilación de información y los conocimientos y percepciones de expertos locales y científicos en diferentes áreas.

La vulnerabilidad de los servicios ecosistémicos en el AMP Uramba es relativamente baja. Es alta en la región exterior (La Barra, Ladrilleros, Juanchaco) y baja en la región interior (Quebrada Valencia, Agujeros, Luisico). Las amenazas tanto naturales como antropogénicas son más frecuentes en la región exterior. Se encontraron diecisiete factores de estrés, entre ellos, el aumento del nivel del mar es el principal y vulnera la mayoría de los servicios ecosistémicos del AMP Uramba.

El aumento del nivel del mar podría afectar la protección costera en playas de arena, planos lodosos y manglares; la regulación del clima en playas de arena y planos lodosos; la recreación y el turismo en playas de arena y manglares; el mantenimiento del ciclo de vida en manglares, y la provisión de alimentos en planos lodosos.

Los métodos presentados para evaluar la vulnerabilidad de los SE están en desarrollo. Los resultados de este estudio se suman a los obtenidos mediante enfoques similares. Estos resultados muestran que la combinación de datos cuantitativos con el conocimiento de expertos y datos cualitativos con el conocimiento ecológico tradicional es un método valido para realizar evaluaciones de áreas marinas ocean water decrease its impact and the vulnerability to this threat. Similar outcomes have been identified in the Baltic Sea (Griffiths et al., 2017), meaning that the system as a whole exhibits a great recovery potential, even when it is heavily perturbed (Mumby et al., 2014).

\section{CONCLUSIONS}

After gathering the knowledge and perceptions of experts in different areas, who thanks to their expertise and knowledge of Uramba MPA were able to identify and map the ES offered and their stressors, and their vulnerability.

The vulnerability of ecosystem services in Uramba MPA is relatively low. It is high at the outer region (La Barra, Ladrilleros, Juanchaco) and low at the inner region (Quebrada Valencia, Agujeros, Luisico). In the same way, both natural and anthropogenic stressors are more frequent at the outer region. From the seventeen stressors identified, sea level rise is the main stressor that vulnerate Uramba MPA's ecosystems services.

Sea level rise is the main potential stressor among Uramba MPA's ecosystem services. It could affect coastal protection in sandy beaches, mud flats, and mangroves; climate regulation in sandy beaches and mud flats; recreation and tourism in sandy beaches and mangroves: life cycle maintenance in mangroves, and food provision in mud flats.

The methods presented to assess the vulnerability of ES are in development. Results from this study add to those achieved through similar approaches. These results show that combining quantitative data with expert knowledge and community participation in these assessments is highly effective. The authors appreciate the ideas of local communities, researchers and entities that can contribute to the understanding of the vulnerability of MPAs and their ES.

Although the protection of Uramba MPA and the presence of environmental authorities has promoted good management to the coastal and marine ecosystems and biotic resources, the results obtained in this research can guide decisions-makers in the understanding of the ecosystems in Uramba MPA, and can aid in the creation or reinforcing of management and conservations tools as the Marine Protected Areas subsystems' Action Plan, the Uramba MPA National Natural Park Management Plan, the La Sierpe Regional Natural Park Management Plan, 
protegidas. Los autores agradecen las ideas de las comunidades locales, investigadores y entidades administrativas que puedan contribuir al entendimiento de la vulnerabilidad de las AMP y sus SE.

A pesar de que el manejo del AMP Uramba y la presencia de autoridades ambientales han promovido un buen manejo de los ecosistemas marinos y costeros, los resultados obtenidos en esta investigación pueden orientar a los tomadores de decisiones en la comprensión de los ecosistemas en el AMP Uramba y ayudar en la creación o fortalecimiento de herramientas de manejo y conservación como el plan de acción de los Subsistemas de Áreas Marinas Protegidas, el plan de manejo del Parque Nacional Natural Uramba, el plan de manejo del Parque Natural Regional La Sierpe y el plan de manejo del Distrito Integrado Manejado de La Plata.

\section{AGRADECIMIENTOS}

A Colciencias por el financiamiento del proyecto. A la Base Naval ARC Málaga por su apoyo. A los consejos y miembros de las comunidades por el aporte de valiosos conocimientos locales y su hospitalidad. $\mathrm{Al}$ Instituto Colombiano de Investigaciones Marinas y Costeras "José Benito Vives de Andreis" por permitir el desarrollo del taller de expertos durante el Curso de Manejo Integrado de Zonas Costeras en 2012 y por su apoyo durante el estudio. Al Ministerio de Ambiente y Desarrollo Sostenible de Colombia por su apoyo durante el estudio. A Idea Wild por el equipo de subvención para desarrollar esta investigación. A Lara Birkart por su constructiva retroalimentación sobre la mejora de este manuscrito. A Emily Pappo por su revisión de escritura en inglés. A Juan Carlos Mejía por su apoyo en la construcción del mapa de ecosistemas del AMP Uramba. and La Plata Managed Integrated District Management Plan.

\section{AKNOWLEDGEMENTS}

To Colciencias for funding the project. To the Navy Base ARC Málaga for their support. To communities' councils and members for their contribution of valuable traditional ecological knowledge, and their hospitality. To the Colombian Marine and Coastal Research Institute "Jose Benito Vives de Andreis" for allowing the expert's workshop development during the Integrated Coastal Zone Management Course in 2012 and for their support during the study. To the Environment and Sustainable Development Ministry of Colombia for their support during the study. To Idea Wild for the grant equipment to develop this research. To Lara Birkart for her constructive feedback to the improvement of this manuscript. To Emily Pappo for her English writing revision. To Juan Carlos Mejia for his support on construct the Uramba MPA ecosystems map.

\section{BIBLIOGRAFÍA/LITERATURE CITED}

Agardy, T., J. Alder, P. Dayton, S. Curran, A. Kitchingman, M. Wilson, A. Catenazzi, J. Restrepo, C. Birkeland, S. Blaber, S. Saifullah, G. Branch, D. Boersma, S. Nixon, P. Dugan, N. Davidson, and C. Vorosmarty. 2005. Coastal Systems. 513-549. In: Hassan, R., R. Scholes and N. Ash (Eds.). Ecosystems and human well-being: current state and trends, Volume 1. Findings of the condition and trends working group of the Millennium Ecosystem Assessment. Millennium Ecosystem Assessment. Series 1. Island Press, Washington. 47 p.

Aldana-Domínguez, J., C. Montes, M. Martínez, N. Medina, J. Hahn, and M. Duque. 2017. Biodiversity and ecosystem services knowledge in the Colombian Caribbean: progress and challenges. Tropical Conservation Science, 10: 1-41. doi.org/10.1177/1940082917714229

Alongi, D.M. 2008. Mangrove forests: resilience, protection from tsunamis, and responses to global climate change. Estuar Coast Shelf Sci., 76: 1-13.

Backer, H., J.M. Leppänen, A.C. Brusendorff, K. Forsius, M. Stankiewicz, J. Mehtonen, and T. Haaranen. 2010. HELCOM Baltic Sea Action Plan: a regional programme of measures for the marine environment based on the ecosystem approach. Mar Pollut. Bull., 60(5): 642-649. 
Baho, D.L., C.R. Allen, A.S. Garmestani, H.B. Fried-Petersen, S.E. Renes, L. Gunderson, and D.G. Angeler. 2017. A quantitative framework for assessing ecological resilience. Ecol. Soc., 22(3): 17. https://doi.org/10.5751/ES-09427-220317

Barbier, E.B., S.D. Hacker, C. Kennedy, and E.W. Koch. 2011. The value of estuarine and coastal ecosystem services. Ecol. Monogr., 81(2): 169-193.

Blanco, J.F., E.A. Estrada, L.F. Ortiz, and L.E. Urrego. 2012. Ecosystem-wide impacts of deforestation in mangroves: the Urabá Gulf (Colombian Caribbean) case study. Int. Sch. Res. Notices, 1-14. doi.org/10.5402/2012/958709

Boyd, J. and S. Banzhaf. 2007. What are ecosystem services? The need for standardized environmental accounting units. Ecol. Econ., 63 (2-3): 616-626.

Branch, G., R.C. Thompson, T. P Crowe, J.C. Castilla, O. Langmead and S. Hawkins. 2008. Rocky intertidal shores: Prognosis for the future, In: Polunin, N.V.C. (Ed.), Aquatic Ecosystems: Trends and Global Prospects, 209-225. https://doi.org/10.1017/CBO9780511751790.020

Cantera, J.R. 1991. Etude structurale des mangroves et des peuplements littoraux des deux baies du Pacifique Colombien (Málaga et Buenaventura). Rapport avec les conditions du milieu et les peerturbations anthropiques. These d'Etat Sciences. Université d'Aix-Marseille II, Marseille. 429 p.

Cantera, J.R. 2016. Bioerosion patterns in Tertiary rock cliffs of the Pacific coast of Colombia (Tropical Eastern Pacific). Rev. Acad. Colomb. Cienc. Ex. Fis. Nat., 40(155): 288-299. http://dx.doi.org/10.18257/r

Cantera, J.R., R. Neira, and C. Ricaurte. 1998. Bioerosión en la costa Pacífica de Colombia. Fondo José Celestino Mutis. FEN y Tercer Mundo, Bogotá. $133 \mathrm{p}$.

Cantera, J.R., B.A. Tomassin and P. Arnaud. 1999. Faunal zonation and assemblages in the Pacific Colombian mangroves. J Hydrol, 413: 17-33.

Cantera, J.R., E. Londoño-Cruz, L.M. Mejía-Ladino, L. Herrera-Orozco, C. Satizabal, and N. Uribe- Castañeda. 2013. Environmental Issues of a marine protected area in a tectonic estuary in the Tropical Eastern Pacific: Uramba (Malaga Bay Colombia): context, biodiversity, threats and challenges. Water Resour. Res., 5: 1037-1047. https://doi.org/10.4236/jwarp.2013.511109

Castellanos-Galindo, G.A., J.R Cantera, S. Espinosa and L.M. Mejía-Ladino. 2011. Use of local ecological knowledge. scientist's observations and grey literature to assess marine species at risk in a Tropical Eastern Pacific Estuary. Aquatic Conserv: Mar. Freshw. Ecosyst., 21: 37-48.

Chambers, L.G., S.E. Davis, T. Troxler, A. Downey-Wall, J.N. Boyer and L.J. Scinto. 2013. Biogeochemical effects of simulated sea level rise on carbon loss in an Everglades mangrove peat soil. J Hydrol., 726: 195-211. https://doi.org/10.1007/s10750-013-1764-6

Cobo-Viveros, A.M. 2007. Medición de volúmenes de bioerosión debidos a perforación y fauna asociada a este proceso en dos acantilados rocosos terciarios de la Bahía de Buenaventura, Pacifico colombiano. Tesis preg. Biol., Univ. Valle, Cali. 128 p.

Cobo-Viveros, A.M. and J.R. Cantera-Kintz. 2015. Main factors determining bioerosion patterns in rocky cliffs in a drowned valley estuary in the Colombian Pacific (Tropical Eastern Pacific). Geomorphology, 246: 220-231.

Coll, M., A.C. Fonseca y J. Cortés. 2001. El manglar y otras asociaciones vegetales de la laguna de Gandoca. Limón, Costa Rica. Rev. Biol. Trop., 49(2): 321-329

Cork, S. 2010. Resilience of social-ecological systems. In: Resilience and transformation: preparing Australia for uncertain futures. Csiro, Melbourne. $205 \mathrm{p}$.

Costanza, R., R. de Groot, M. Grasso, and B. Hannon. 1997. The value of the world's ecosystem services and natural capital. Nature 387(15): 253-260. https://doi.org/10.1016/S0921-8009(98)00020-2

Costanza, R., R. de Groot, P. Sutton, S. van der Ploeg, J. Anderson, I. Kubiszewski, S. Farber, R.K. Turner. 2014. Changes in the global value of ecosystem services. Global Environ. Chang., 26: 152-158.

Costanza, R., R. de Groot, L. Braat, I. Kubiszewski, L. Fioramonti, P. Sutton, S. Farber, and M. Grasso. 2017. Twenty years of ecosystem services: How far have we come and how far do we still need to go? Ecosyst. Serv., 28: 1-17. https://doi.org/10.1016/j.ecoser.2017.09.008

Daily, G.C. 1997. Nature's Services-Societal Dependence on Natural Ecosystems. Island Press, Washington D.C.

DANE. 2012. Censo nacional 2005. Proyección de población humana en el municipio de Buenaventura. Valle del Cauca.

Darryl, E., W. Marois and J. Mitsch. 2015. Coastal protection from tsunamis and cyclones provided by mangrove wetlands -a review. Int. J. Biodivers. Sci. Ecosyst. Serv. Manag., 11(1): 71-83. https://doi.org/10.1080/21513732.2014.997292

De Groot, D. 2010. Protecting natural capital for human wellbeing and sustainable development. Science for Environment Policy, DG Environment News Alert, Special Issue Ecosystem Services, 1.

Defeo, O., A. McLachlan, D.S. Schoeman, T.A. Schlacher, J. Dugan, A. Jones, M. Lastra, and F. Scapini. 2009. Threats to sandy beach ecosystems: a review. Estuar. Coast. Shelf Sci., 81: 1-12. 
Dias, M., J. Roma, C. Fonseca, M. Pinto, H.N. Cabral, A. Silva, and C. Vinagre. 2016. Intertidal pools as alternative nursery habitats for coastal fishes. Mar. Biol. Res., 12(4): 331-344. https://doi.org/10.1080/17451000.2016.1143106

Dominati, E.J. 2014. An ecosystem services approach to the quantification of shallow mass movement erosion and the value of soil conservation practices. Ecosyst. Serv., 9: 204-215.

Doody, J.P. 2012. Sand dune conservation, management and restoration. Springer Science and Business Media, Berlin. 306 p.

Duke, N.C., J-O. Meynecke, S. Dittmann, A.M. Ellison, K. Anger, U. Berger, S. Cannicci, K. Diele, K.C. Ewel, C.D. Field, N. Koedam, S.Y. Lee, C. Marchand, I. Nordhaus, and F. Dahdouh-Guebas. 2007. A world without mangroves? Science, 317: 41-42.

FAO. 2010. The state of world fisheries and aquaculture. FAO, Rome. 214 p.

Gislason, H., M. Sinclair, K. Sainsbury and R. O’Boyle, 2000. Symposium overview: incorporating ecosystem objectives within fisheries management. ICES J. Mar. Sci., 57: 468-475.

Gowrie, M.N. 2003. Environmental vulnerability index for the island of Tobago, West Indies. Conserv. Ecol., 7(2): 11-19.

Halpern, B.S., K.L. Mcleod, A.A. Rosenberg, and L.B. Crowder. 2008. Managing for cumulative impacts in ecosystem-based management through ocean zoning. Ocean Coast. Manag., 51(3): 203-211.

Hanley, M.E., S.P.G. Hoggart, D.J. Simmonds, A. Bichot, M.A. Colangelo, F. Bozzeda, and R. Trude. 2014. Shifting sands coastal protection by sand banks, beaches and dunes. Coast. Eng., 87: 136-146.

Invemar-Univalle. 2011. El papel de la salinidad en las asociaciones de larvas de organismos marinos en Bahía Málaga (Pacífico colombiano): valoración de la importancia de esa área como salacuna y su comportamiento con otros estuarios en hábitats tropicales. Informe final. Colciencias-InvemarUnivalle, Cali. 47 p.

Invemar-Univalle-Inciva. 2006. Bases científicas y valoración de la biodiversidad marina y costera de Bahía Málaga (Valle del Cauca), como uno de los instrumentos necesarios para que sea considerada un área protegida. Informe final. Colciencias-Invemar-Univalle-Inciva, Cali. 813 p.

IPCC. 2019. Refinement to the 2006 IPCC guidelines for national greenhouse gas inventories. Intergovernmental Panel on Climate Change. 672 p.

Kaly, U., L. Briguglio, H. McLeod, S. Schmall, C. Pratt, and R. Pal. 1999. Environmental Vulnerability Index (EVI) to summarise national environmental vulnerability profiles. SOPAC, Suva. $67 \mathrm{p}$.

King, P.G. and D. Symes. 2004. Potential loss in GNP and GSP from a failure to maintain California's beaches. Shore Beach, 72(1): 3-7.

King, P.G., C. Nelsen, J.E. Dugan, D.M. Hubbard, and K.L. Martin. 2018. Valuing beach ecosystems in an age of retreat. Shore Beach, 86(4): 45-59.

Laurans, Y., N. Pascal, T. Binet, L. Brander, E. Clua, G. David, D. Rojat, and A. Seidl. 2013. Economic valuation of ecosystem services from coral reefs in the South Pacific: taking stock of recent experience. Journal Environ. Manag., 116: 135-144.

Liquete, C., C. Piroddi, E.G. Drakou, L. Gurney, S. Katsanevakis, A. Charef, and B. Egoh. 2013. Current status and future prospects for the assessment of marine and coastal ecosystem services: a systematic review. Plos One, 8(7), e67737. https://doi.org/10.1371/journal.pone.0067737

Marcelli, M., S. Scani, F. Manfredi, E. Mancini, and F. Carli. 2018. A benthic zonation system as a fundamental tool for natural capital assessment in a marine environment: a case study in the Northern Tyrrhenian Sea, Italy. Sustainability, 10(3786): 1-15. https://doi.org/10.3390/su10103786

Mazda, Y., M. Magi, Y. Ikeda, T. Kurokawa, and T. Asano. 2006. Wave reduction in a mangrove forest dominated by Sonneratia sp. Wetl Ecol. Manag., 14: 365-378.

McCartney, M., M. Finlayson, and S. de Silva. 2015. Sustainable development and ecosystem services. Brief for GSDR 2015.

Metcalf, S.J., E.I. Van Putten, S. Frusher, N.A. Marshall, M. Tull, N. Caputi, M. Haward, A.J. Hobday, N.J. Holbrook, S.M. Jennings, G.T. Pecl and J.L. Shaw. 2015. Measuring the vulnerability of marine social-ecological systems: a prerequisite for the identification of climate change adaptations. Ecol. Soc., 20(2): 35. http://dx.doi.org/10.5751/ES-07509-200235

Moberg, F. and C. Folke. 1999. Ecological goods and services of coral reef ecosystems. Ecol. Econ., 29(1): 215-233.

Mooney, H.A. and P.R. Ehrlich. 1997. Ecosystem services: a fragmentary history. 11-19. In G.C. Daily (Ed.). Nature's Services: societal dependence on natural ecosystems. Island Press, Washington, DC.

Mukherjee, N., W.J. Sutherland, L. Dicks, and J. Huge. 2014. Ecosystem service values for mangroves in Southeast Asia: a meta-analysis and value transfer application ecosystem service valuations of mangrove ecosystems to inform decision making and future valuation exercises. Plos One, 9(9): e107706. https://doi.org/10.1371/journal.pone.0111386 
Mumby, P., I. Chollett, Y. Bozec and N.H. Wolff. 2014. Ecological resilience, robustness and vulnerability: how do these concepts benefit ecosystem management? Curr. Opin. Environ. Sustain., 7: 22-27.

Olivo, M.L., A. Martin, V. Saez-Saez, and A. Soto. 2011. Vulnerabilidad al incremento del nivel del mar: pérdida de tierra en el área, cabo Codera-Laguna de Tacarigua, estado Miranda, Venezuela. Terra Nueva Etapa, 27: 125-145.

Oppenheimer, M., M. Campos, R. Warren, J. Birkmann, G. Luber, B. O’Neill and K. Takahashi. 2014. Emergent risks and key vulnerabilities. $1039-1099$. In: Climate Change 2014: Impacts, Adaptation, and Vulnerability. Part A: Global and Sectoral Aspects. Contribution of Working Group II to the Fifth Assessment Report of the Intergovernmental Panel on Climate Change. Cambridge University Press, Cambridge, United Kingdom and New York.

Osland, M.J., N.M. Enwright, R.H. Day, C.A. Gabler, C.L. Stagg, and J.B. Grace. 2016. Beyond just sea-level rise: considering macroclimatic drivers within coastal wetland vulnerability assessments to climate change. Glob. Change Biol., 22: 1-11. https://doi.org/10.1111/gcb.13084

Palacios, M.L. and J.R. Cantera. 2017. Mangrove timber use as an ecosystem service in the Colombian Pacific. Hydrobiologia, 803(1): 345-358.

Palacios, M.L., Cantera J.R., and Peña E. J. 2019. Carbon stocks in mangrove forests of the Colombian Pacific. Estuar. Coast. Shelf Sci., $227:$ 1-7.

Posada, B.O.P., W.H. Pineda y G.G. Ospitia. 2009. Diagnóstico de la erosión y sedimentación en la zona costera del Pacífico colombiano. Invemar, Santa Marta. 148 p.

Riascos, J.M., N. Valencia, E.J. Peña, and J.R. Cantera. 2019. Inhabiting the technosphere: the encroachment of anthropogenic marine litter in Neotropical mangrove forests and its use as habitat by microbenthic biota. Mar. Pollut. Bull., 142: 559-568.

Robertson, H.A. and T.K. McGee. 2003. Applying local knowledge: the contribution of oral history to wetland rehabilitation at Kanyapella Basin, Australia. J. Environ. Manag., 69(3): 275-87.

Rojas, A.M., C.A. Ruiz-Agudelo, M.C. Diazgranados, H. Polanco, and R. Anderson, R. 2019. Approach to an integral valuation of mangrove's ecosystem services in a marine protected area. Colombian Pacific region. Journal Environ. Econ. Policy, 1-21. https://doi.org/10.1080/21606544.2019.1584127

Rubio, E. 1984. Preliminary taxonomic study of Bahía Malaga’s, Colombia Icthyofauna. Cespedesia, 13(47-48): 97-111.

Sasaki, T., T. Furukawa, Y. Iwasaki, M. Seto, M., and A.S. Mori. 2015. Perspectives for ecosystem management based on ecosystem resilience and ecological thresholds against multiple and stochastic disturbances. Ecol. Indic., 57: 395-408.

Schröter, D., M.J. Metzger, W. Cramer, and R. Leemans. 2004. Vulnerability assessment-analysing the human-environment system in the face of global environmental change. ESS Bulletin, 2(2): 11-17.

Schumacher, E. 1973. Small is beautiful. New York: Harper Torchbooks.

Simm, J. 1996. Beach Management Manual. CIRIA, London. 233 p.

Spalding, M. and C.L. Parrett. 2019. Global patterns in mangrove recreation and tourism. Mar. Policy, 110: 103540. https://doi.org/10.1016/j. marpol.2019.103540

Spears, B., S.C. Ives, D.G. Angeler, C.R. Allen, S. Birk, L. Carvalho, S. Cavers, F. Daunt, R.D. Morton, M.J.O. Pocock, G. Rhodes, and S.J. Thackeray. 2015. Effective management of ecological resilience - are we there yet? J. Appl. Ecol. https://doi.org/10.1111/1365-2664.12497

Steinmuller, H.E. and L.G. Chambers. 2019. Characterization of coastal wetland soil organic matter: implications for wetland submergence. Sci. Total Environ, 677: 648-659. https://doi.org/10.1016/j.scitotenv.2019.04.405

Thompson, B.S. and S.M. Rog. 2019. Beyond ecosystem services: using charismatic megafauna as flagship species for mangrove forest conservation. Environ. Sci. Policy, 102: 9-17.

Turner II, B.L., R.E. Kasperson, P.A. Matson, J.J. Mccarthy, R.W. Corell, L. Christensen, N. Eckley, J.X. Kasperson, A. Luers, M.L. Martello, C. Polsky, A. Pulsipher, and A. Schiller. 2003. A framework for vulnerability analysis in sustainability science. PNAS, 100(14): 8074-8079.

Univalle. 2010. Environmental and social pre-feasibility study on the construction of a deepwater port in Uramba. Pacífico colombiano. Final report. Univalle, Cali. 290 p.

Veidemane, K. 2019. Contribution of ecosystem services to achievement of the sustainable development goals. Proceedings 2019, 30(1): 8. https://doi. org/10.3390/proceedings2019030008

Violante, C. 2009. Rocky coast: geological constraints for hazard assessment. Geol. Soc. Spec. Publ., 322(1): 1-31. https://doi.org/10.1144/SP322.1

Vo, Q.T., C. Kuenzer, Q.M. Vo, F. Moder, and N. Oppelt. 2012. Review of valuation methods for mangrove ecosystem services. Ecol. Indic., 23: $431-446$. 
Weißhuhn, P., F. Müller, and H. Wiggering. 2018. Ecosystem vulnerability review: proposal of an interdisciplinary ecosystem assessment approach. Environ. Manag., 61: 904-915. https://doi.org/10.1007/s00267-018-1023-8

Wilson, J.G. 2005. The intertidal ecosystem: The value of Ireland's shores. Royal Irish Academy. 203 p.

Wood, S.J.R., S.K. Jones, J.A. Jhonson, K.A. Brauman, R. Chaplin-Kramer, A. Fremier, E. Girvetz, L.J. Gordon, C.V. Kappel, L. Mandle, M. Mulligan, P. O'Farrel, W.K. Smith, L. Willemen, W. Zhang, and F.A. DeClerck. 2018. Distilling the role of ecosystem services in the Sustainable Development Goals. Ecosyst. Serv., 29: 70-82.

Woodhead, A.J., C.C. Hicks, A.V. Norström, G.J. Gareth, and N.J. Graham. 2019. Coral reef ecosystem services in the Anthropocene. Funct. Ecol., 33(6): 1023-1034.

Yáñez-Arancibia, A., R.R. Twilley, and A.L. Lara-Domínguez. 1998. Los ecosistemas de manglar frente al cambio climático global. Madera Bosques, 4(2): 3-19.

Zacharias, M.A. and E.J. Gregr. 2005. Sensitivity and Vulnerability in Marine Environments: an approach to identifying vulnerable marine areas. Conserv. Biol., 19(1): 86-97.

RECIBIDO/RECEIVED: 26/07/2020

ACEPTADO/ACCEPTED: 28/10/2020 